Article

\title{
Distributed Secondary Control for Islanded Microgrids Cluster Based on Hybrid-Triggered Mechanisms
}

\author{
Shengxuan Weng ${ }^{1, *(1)}$, Yusheng Xue ${ }^{2}$, Jianbo Luo ${ }^{2}$ and Yanman $\mathrm{Li}^{2}$ \\ 1 Institute of Advanced Technology, Nanjing University of Posts and Telecommunications, Nanjing 210023, China \\ 2 NARI Technology Co., Ltd., Nanjing 210023, China; xueyusheng@sgepri.sgcc.com.cn (Y.X.); \\ luojianbo@sgepri.sgcc.com.cn (J.L.); liyanman@sgepri.sgcc.com.cn (Y.L.) \\ * Correspondence: shxweng@gmail.com
}

Received: 20 February 2020; Accepted: 17 March 2020; Published: 23 March 2020

check for updates

\begin{abstract}
Considering the communication resources limitation, the hybrid-triggered mechanism based distributed control of islanded microgrids cluster is proposed, which can restore the frequency to the rated value and realize the active power sharing when the disturbance occurs. The hybrid-triggered mechanism consists of the self- and event-triggered mechanisms, which are configured at each leader and follower distributed generation to determine the inter-microgrids and intra-microgrid information transmission, respectively. The communication burdens can be sharply reduced since the information is transmitted aperiodically only when the proposed triggering conditions are satisfied under the hybrid-triggered mechanism. Moreover, Zeno behavior is analyzed to be avoided to make the hybrid-triggered mechanism reasonable and practicable for practical islanded microgrids cluster. The simulation verifies the effectiveness of theoretical results.
\end{abstract}

Keywords: distributed control; triggered mechanism; frequency restoration; power sharing; islanded microgrids cluster

\section{Introduction}

Due to the properties of low environmental costs, renewability and worldwide distribution, the distributed generation (DG) has been used widely in recent years. This brings the new change and challenge to the traditional power system [1,2], and leads to the birth of microgrid (MG) which is a low voltage power system containing DGs, loads, and storage units [3,4]. In order to enable the maximum utilization of renewable sources and suppress the stress and aging of the components in MG, multiple MGs interlink with each other to constitute the MGs cluster [5].

When the MGs cluster operates in the islanded mode, the frequency should be maintained to the rated value and the active power demands should be dispatched properly among the DGs by constructing the proper primary and secondary control method. The primary control stabilizes the MGs cluster and establishes the active power sharing based on droop control idea, and the secondary control compensates the frequency deviations caused by the primary control [6-10], achieves the power flow balance among multiple MGs, and increases the reliability of the whole islanded MGs cluster by dispatching power among MGs [11]. Different from the primary control based on decentralized architecture, the implementation of secondary control requires the information transmission among the DGs, and it has been constructed based on distributed architecture usually, where each DG executes the secondary control based on its own and the neighboring DGs' information. In [12], the $P / Q$ control mode was adopted to construct a clustering and cooperative control strategy for MGs cluster, and a distributed power management scheme based on the droop operating principles for MGs 
cluster was considered in [13]. A cluster-oriented distributed cooperative control strategy for MGs cluster was studied in [14] to achieve the frequency restoration and power sharing under a two-layer communication network, and the issue of intermittent communication was further concerned in [11].

As mentioned, most of the existing distributed secondary control methods require that the information should be transmitted continuously among the DGs. This is not suitable for the modern information technology since the information transmission is realized discretely by using wired/wireless digital communication. Although the continuous information transmission can be executed discretely in practical application according to the periodic sampled-data scheme, it increases the communication burdens and wastes the communication resources since the fixed sampling rate is selected conservatively under the worst case. The event-triggered and self-triggered mechanisms used widely in networked control systems $[15,16]$ and multi-agent systems $[17,18]$ should be the effective methods to address this issue. The event-triggered mechanism continuously monitors the proposed triggering condition based on a system's state. An event is triggered when the triggering condition is satisfied, and the information is sampled and broadcast at each triggering time instant. In [19], the distributed optimization control law was constructed based on an event-triggered mechanism to achieve the optimal power outputs on generators and optimal electricity usage on loads. In [20], the event-triggered mechanism was introduced in the secondary restoration control of isolated microgrid to reduce the inter-agent communication. The distributed event-triggered mechanism for economic dispatch in power grid was considered in [21], and the power sharing control under event-triggered mechanism was considered in [22]. In [23], the event-triggered mechanism is introduced in the distributed optimal frequency regulation of multiple distributed power generations. On the other hand, the self-triggered mechanism precomputes the next triggering time instant based on predictons using previously received data and knowledge of system's state at the present triggering time instant. In [24], the self-triggered mechanism was integrated in the microgrid coordination control for DGs to reduce the communication packages and save the usage of bandwidth. In [25], the distributed self-triggered $P-Q$ control for power balance in microgrid was studied. As mentioned, both of the event- and self-triggered mechanisms can reduce the communication burdens since the period of information transmission is adjusted adaptively according to a system's state.

Although there exists some research concerned with the event- or self-triggered mechanism based control of the microgrid, the topic about distributed secondary control for islanded MGs cluster is still lacking to the best of the authors' knowledge, and this motivates the current study. The hybrid-triggered mechanism, which includes the self-triggered mechanism for inter-MGs communication and the event-triggered mechanism for intra-MG communication, is introduced in the distributed secondary control of islanded MGs cluster in this paper. It aims to reduce the communication burdens while performing the distributed secondary control to restore the frequency and realize the active power sharing in islanded MGs cluster. The main contributions of this paper are summarized as follows:

1. The inter-MGs distributed control, where the information is transmitted through the inter-MGs communication network, is configured at the leader DG in each MG. It aims to achieve the cooperation among multiple MGs in the cluster. The self-triggered mechanism is introduced in the inter-MGs distributed control to reduce the inter-MGs communication burdens while achieving the frequency restoration and active power sharing of all leader DGs in finite-time.

2. The intra-MG distributed control, where the information is transmitted through the intra-MG communication network, is configured at the follower DG. It aims to achieve the cooperation within each MG by driving the frequency and active power output ratio of follower DGs to those of leader DG, respectively. The event-triggered mechanism is introduced in the intra-MG distributed control to decrease the information amount transmitted in the intra-MG communication network. 
3. The hybrid-triggered mechanism based distributed secondary control integrates the self-triggered inter-MGs and event-triggered intra-MG distributed control, and it can realize the frequency restoration and active power sharing of the whole islanded MGs cluster. Furthermore, Zeno behavior is analyzed to be avoided, which demonstrates the rationality and practicability of the hybrid-triggered mechanism in practical islanded MGs cluster application.

This paper is organized as follows. Section 2 describes the islanded MGs cluster configuration and control purposes. The hybrid-triggered mechanism based distributed secondary control for islanded MGs cluster is constructed and analyzed in Section 3. Section 4 represents the simulation result to show the effectiveness of theory, and the conclusion is finally stated in Section 5.

\section{Problem Formulation}

\subsection{Islanded MGs Cluster Configuration}

Consider the islanded MGs cluster including $s$ MGs labeled as $M G_{1}, M G_{2}, \ldots, M G_{s}$, respectively, and each $M G_{l}(l=1, . ., s)$ contains one leader DG labeled as $D G_{l, 0}$ and $n^{l}$ follower DGs denoted as $D G_{l, 1}, \ldots, D G_{l, n^{l}}$ respectively. The information is transmitted among DGs in the whole islanded MGs cluster through a double-layer communication network shown in Figure 1 to execute the distributed secondary control.

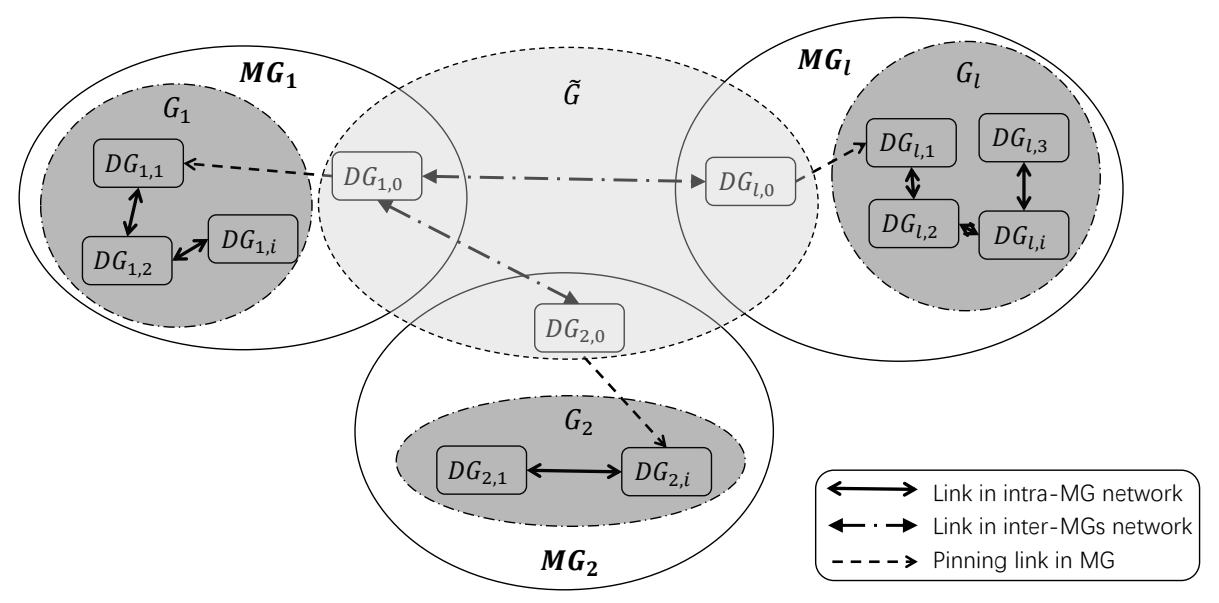

Figure 1. An example of double-layer communication network configuration in islanded MGs cluster.

The information transmission between MGs is implemented through the inter-MGs communication network whose topology can be described by the graph $\tilde{\mathcal{G}}=(\tilde{\mathcal{V}}, \tilde{\mathcal{E}})$. The node set $\tilde{\mathcal{V}}=\left\{D G_{1,0}, D G_{2,0} \ldots, D G_{s, 0}\right\}$ containing all the leader DGs in cluster, and the edge set $\tilde{\mathcal{E}}=\left\{\left(D G_{m, 0}, D G_{l, 0}\right) \mid\right.$ if $\left.D G_{m, 0} \rightarrow D G_{l, 0}\right\}$ where the symbol $D G_{m, 0} \rightarrow D G_{l, 0}$ means that the information of $D G_{m, 0}$ can be transmitted to $D G_{l, 0}$ directly through communication network $\tilde{\mathcal{G}}$. The adjacency matrix of $\tilde{\mathcal{G}}$ is denoted as $\tilde{A}=\left\{\tilde{a}_{m l}\right\} \in R^{s \times s}$, where $\tilde{a}_{m l}=1$ if and only if $\left(D G_{m, 0}, D G_{l, 0}\right) \in \tilde{\mathcal{E}}$; otherwise, $\tilde{a}_{m l}=0$, and $\tilde{a}_{l l}=1$. The Laplacian matrix of graph $\tilde{\mathcal{G}}$ is given as $\tilde{L}=\operatorname{diag}\left\{\sum_{m=1}^{s} \tilde{a}_{1 m}, \ldots, \sum_{m=1}^{S} \tilde{a}_{s m}\right\}-\tilde{A}$, which is semi-positive definite since the graph $\tilde{\mathcal{G}}$ is assumed to be undirected and connected [26]. The neighboring set of $D G_{l, 0}$ in the communication network $\tilde{\mathcal{G}}$ is defined as $\tilde{N}_{l}=\left\{D G_{m, 0} \in \tilde{\mathcal{V}} \mid\left(D G_{m, 0}, D G_{l, 0}\right) \in \tilde{\mathcal{E}}\right\}$, and its cardinal number is $\left|\tilde{N}_{l}\right|$.

The information transmission within each $M G_{l}(l=1, \ldots, s)$ is implemented through the intra-MG communication network $\mathcal{G}^{l}=\left(\mathcal{V}^{l}, \mathcal{E}^{l}\right)$, where the node set $\mathcal{V}^{l}=\left\{D G_{l, 1}, D G_{l, 2} \ldots, D G_{l, n^{l}}\right\}$ containing all the follower $D G$ in $M G_{l}$ and the edge set $\mathcal{E}^{l}=\left\{\left(D G_{l, j}, D G_{l, i}\right) \mid\right.$ if $\left.D G_{l, j} \rightarrow D G_{l, i}\right\}$. Similarly, the adjacency and Laplacian matrix of $\mathcal{G}^{l}$ are denoted as $A^{l}=\left\{a_{i j}^{l}\right\} \in R^{n^{l} \times n^{l}}$ and $L^{l}=\operatorname{diag}\left\{\sum_{j=1}^{n^{l}} a_{1 j}, \ldots, \sum_{j=1}^{n^{l}} a_{n^{l} j}\right\}-A^{l}$, 
respectively. The neighboring set of $D G_{l, i}$ in the communication network $\mathcal{G}^{l}$ is $N_{i}^{l}=\left\{D G_{l, j} \in \mathcal{V}^{l} \mid\left(D G_{l, j}, D G_{l, i}\right) \in \mathcal{E}^{l}\right\}$ with the cardinal number $\left|N_{i}^{l}\right|$. It is also assumed that the graph $\mathcal{G}^{l}$ is undirected and connected. Moreover, at least one follower $D G_{l, i}$ can receive the information of the leader $D G_{l, 0}$ in each $M G_{l}(l=1, \ldots, s)$.

\subsection{Control Purposes}

For the $i$ th $D G$ in $M G_{l}\left(l=1, \ldots, s, i=0, \ldots, n^{l}\right)$, i.e., $D G_{l, i}$, the active power output is adjusted by the primary control (1) based on the $P-f$ droop characteristic [14]

$$
\omega_{l, i}(t)=\omega_{l, i}^{\text {nom }}(t)-K_{l, i} P_{l, i}(t)
$$

where $\omega_{l, i}$ and $P_{l, i}$ are the frequency and active power output of $D G_{l, i}$, respectively, the positive constant $K_{l, i}$ is the droop coefficient, and $\omega_{l, i}^{\text {nom }}$ denotes the frequency nominal set-point.

As mentioned, the primary control (1) leads to the unpredictable and unacceptable deviations of frequency from their reference values, and the secondary control should be constructed to regulate the frequency nominal set-point $\omega_{l, i}^{\text {nom }}$ to address this issue. According to Equation (1), the set-point $\omega_{l, i}^{\text {nom }}$ can be regulated as follows shown in [14]

$$
\omega_{l, i}^{n o m}=\int\left(u_{l, i}^{\omega}+K_{l, i} u_{l, i}^{P}\right) d t
$$

where the auxiliary frequency control $u_{l, i}^{\omega}=\frac{d}{d t} \omega_{l, i}(t)$ and the auxiliary active power control $u_{l, i}^{P}=\frac{d}{d t} P_{l, i}(t)$. The secondary control should be designed to regulate $u_{l, i}^{\omega}$ and $u_{l, i}^{P}$ in Equation (2) to accomplish the following two purposes.

Purpose 1: Restore the frequency. namely,

$$
\left|\omega_{l, i}-\omega^{\text {rated }}\right| \leq \bar{\varepsilon}_{\omega}
$$

for any $i \in\left\{0, \ldots, n^{l}\right\}$ and $l \in\{1, \ldots, s\}$, where the constant $\omega^{\text {rated }}$ is the rated frequency of islanded MGs cluster, and the positive constant $\bar{\varepsilon}_{\omega}$ is the acceptable fluctuation range. This purpose is the basis of safe and stable operation of islanded MGs cluster.

Purpose 2: Realize the active power sharing. Equivalently,

$$
\left|\frac{P_{l, i}}{P_{l, i}^{\text {max }}}-\frac{P_{m, j}}{P_{m, j}^{\max }}\right| \leq \bar{\varepsilon}_{P}
$$

for any $i \in\left\{0, \ldots, n^{l}\right\}, j \in\left\{0, \ldots, n^{m}\right\}$ and $l, m \in\{1, \ldots, s\}$, where the positive constant $P_{l, i}^{\max }$ is the instantaneous maximum capacity of the active power of $D G_{l, i}$, and $\bar{\varepsilon}_{P}$ is the acceptable fluctuation range. This purpose ensures that all the DGs in the MGs cluster share the entire loads fairly, which could suppress stress and aging of the components, and improve the reliability and availability of the MGs cluster [5].

The following distributed secondary inter-MGs control (5) and (6) and intra-MG control (7) and (8) were proposed in [5]

$$
\begin{aligned}
& u_{l, 0}^{\omega}=\sum_{m=1}^{s} \tilde{a}_{l m}\left(\omega_{m, 0}(t)-\omega_{l, 0}(t)\right)+\tilde{a}_{l 0}\left(\omega^{\text {rated }}-\omega_{l, 0}(t)\right) \\
& u_{l, 0}^{P}=\frac{1}{K_{l, 0}} \sum_{m=1}^{s} \tilde{a}_{l m}\left(K_{m, 0} P_{m, 0}(t)-K_{l, 0} P_{l, 0}(t)\right)
\end{aligned}
$$


where $\tilde{a}_{l 0}=1$ if $D G_{l, 0}$ can receive the information of rated frequency $\omega^{\text {rated }}$; otherwise, $\tilde{a}_{l 0}=0$. It is assumed that at least one $\tilde{a}_{l 0}$ is non-zero for $l=1, \ldots, s$ :

$$
\begin{aligned}
& u_{l, i}^{\omega}=\sum_{i=1}^{n^{l}} a_{i j}^{l}\left(\omega_{l, j}(t)-\omega_{l, i}(t)\right)+a_{i 0}^{l}\left(\omega_{l, 0}(t)-\omega_{l, i}(t)\right) \\
& u_{l, i}^{P}=\frac{1}{K_{l, i}}\left(\sum_{i=1}^{n^{l}} a_{i j}^{l}\left(K_{l, j} P_{l, j}(t)-K_{l, i} P_{l, i}(t)\right)+a_{i 0}^{l}\left(K_{l, 0} P_{l, 0}(t)-K_{l, i} P_{l, i}(t)\right)\right)
\end{aligned}
$$

where $a_{i 0}^{l}=1$ if $D G_{l, i}$ can receive the information of the leader $D G_{l, 0}$; otherwise, $a_{i 0}^{l}=0$. At least one $a_{i 0}^{l}$ is non-zero for $i=1, \ldots, n^{l}$ as illustrated in Section 2.1. Setting the droop coefficients as $K_{l, i} P_{l, i}^{\max }=K_{m, j} P_{m, j}^{\max }$ for all $i \in\left\{0, \ldots, n^{l}\right\}, j \in\left\{0, \ldots, n^{m}\right\}$ and $l, m \in\{1, \ldots, s\}$ in control law (5)-(8) achieves Purposes 1 and 2 as shown in [5].

However, it should be mentioned that the implementation of control law (5)-(8) requires each DG to transmit its own information to the neighboring DGs through the communication network continuously, which is not realistic since the information transmission is usually realized discretely by the modern information technology. According to the periodic sampled-data scheme, the continuous information transmission in control (5)-(8) can make the discretization implementation. However, since the fixed sampling rate is selected conservatively under the worst case, the periodic sampled-data scheme increases the communication burdens and wastes the communication resources. To address the problem, the hybrid-triggered mechanism, which includes the self-triggered mechanism for inter-MGs communication and the event-triggered mechanism for intra-MG communication, is introduced in the distributed secondary control of islanded MGs cluster in this paper. The selfand event-triggered mechanisms are configured at leader and follower DG, respectively, to determine the information transmission time instants, and the distributed secondary control based on the triggered information is constructed to achieve Purposes 1 and 2.

\section{Main Result}

Denote the triggering time sequences corresponding to frequency $\omega_{l, i}$ and active power output $P_{l, i}$ of $D G_{l, i}$ as $\left\{t_{0}^{l, i}, t_{1}^{l, i}, \ldots, t_{k}^{l, i}, \ldots\right\}$ and $\left\{\tau_{0}^{l, i}, \tau_{1}^{l, i}, \ldots, \tau_{\tilde{k}}^{l, i}, \ldots\right\}$, respectively, for $i \in\left\{0,1, \ldots, n^{l}\right\}$ and $l \in\{1,2, \ldots, s\}$, where the triggering time instants satisfy $t_{0}^{l, i}<t_{1}^{l, i}<\ldots<t_{k}^{l, i}<\ldots$ and $\tau_{0}^{l, i}<\tau_{1}^{l, i}<\ldots<\tau_{\tilde{k}}^{l, i}<\ldots$ The triggering time instants are determined by a triggered mechanism proposed in the next sections.

\subsection{Self-Triggered Mechanism Based Inter-MGs Distributed Secondary Control}

The inter-MGs distributed secondary control aims to restore the frequency of leader DGs to the rated value $\omega^{\text {rated }}$, and drive the active power output ratio of them to achieve bounded consensus with an acceptable fluctuation range. The self-triggered mechanism is configured at each leader DG in the MGs cluster, which determines the triggering time instants of information transmission among the leader DGs through the inter-MGs communication network.

Self-triggered mechanism: For the leader $D G_{l, 0}$, denoting the last triggering time for frequency $\omega_{l, 0}$ [active power output $P_{l, 0}$ resp.] as $t_{k}^{l, 0}\left[\tau_{\tilde{k}}^{l, 0}\right.$ resp.], $D G_{l, 0}$ polls its neighbors $D G_{m, 0} \in \tilde{N}_{l}$ in inter-MGs communication network $\tilde{\mathcal{G}}$ and collects the neighbors' information $\omega_{m, 0}\left(t_{k}^{l, 0}\right)\left[P_{m, 0}\left(\tau_{\tilde{k}}^{l, 0}\right)\right.$ resp. $]$ at time $t_{k}^{l, 0}\left[\tau_{\tilde{k}}^{l, 0}\right.$ resp.]. Moreover, the next triggering time is calculated at $D G_{l, 0}$ according to Formulas (9) and (10), and the above action will be repeated at $t_{k+1}^{l, 0}\left[\tau_{\tilde{k}+1}^{l, 0}\right.$ resp.]:

$$
\begin{aligned}
& t_{k+1}^{l, 0}=t_{k}^{l, 0}+\max \left\{\frac{\left|f_{l}^{\omega}\left(t_{k}^{l, 0}\right)\right|}{2 \beta^{\omega}\left(2\left|\tilde{N}_{l}\right|+\tilde{a}_{l 0}\right)}, \frac{\varepsilon^{\omega}}{2 \beta^{\omega}\left(2\left|\tilde{N}_{l}\right|+\tilde{a}_{l 0}\right)}\right\} \\
& \tau_{\tilde{\kappa}+1}^{l, 0}=\tau_{\tilde{k}}^{l, 0}+\max \left\{\frac{\left|f_{l}^{P}\left(\tau_{\tilde{\kappa}}^{l, 0}\right)\right|}{4 \beta^{P}\left|\tilde{N}_{l}\right|}, \frac{\varepsilon^{P}}{4 \beta^{P}\left|\tilde{N}_{l}\right|}\right\}
\end{aligned}
$$


where $\beta^{\omega}, \varepsilon^{\omega}, \beta^{P}$, and $\varepsilon^{P}$ are positive constants, the functions

$$
\begin{aligned}
& f_{l}^{\omega}(t)=\sum_{m=1}^{s} \tilde{a}_{l m}\left(\omega_{m, 0}(t)-\omega_{l, 0}(t)\right)+\tilde{a}_{l 0}\left(\omega^{\text {rated }}-\omega_{l, 0}(t)\right) \\
& f_{l}^{P}(t)=\sum_{m=1}^{s} \tilde{a}_{l m}\left(K_{m, 0} P_{m, 0}(t)-K_{l, 0} P_{l, 0}(t)\right)
\end{aligned}
$$

Remark 1. As mentioned, the implementation of self-triggered mechanism (9) and (10) only requires the neighbors' triggered information $\omega_{m, 0}\left(t_{k}^{l, 0}\right)$ and $P_{m, 0}\left(\tau_{\tilde{k}}^{l, 0}\right)$ for $D G_{m, 0} \in \tilde{N}_{l}$, which implies that it satisfies the distributed architecture. Moreover, the mechanism (9) and (10) indicate that the lower bounds of inter-trigger time intervals satisfy $t_{k+1}^{l, 0}-t_{k}^{l, 0} \geq \frac{\varepsilon^{\omega}}{2 \beta^{\omega}\left(2\left|\tilde{N}_{l}\right|+\tilde{a}_{l 0}\right)}$ and $\tau_{\tilde{k}+1}^{l, 0}-\tau_{\tilde{k}}^{l, 0} \geq \frac{\varepsilon^{p}}{4 \beta^{P}\left|\tilde{N}_{l}\right|}$. This means that Zeno behavior, which may cause triggering an infinite number of times in finite time intervals, can be avoided naturally.

Based on the self-triggered mechanism, the inter-MGs distributed secondary control is given as follows:

$$
\begin{aligned}
& u_{l, 0}^{\omega}(t)=\beta^{\omega} \operatorname{sign}_{\varepsilon^{\omega}}\left(f_{l}^{\omega}\left(t_{k}^{l, 0}\right)\right), \text { for } t \in\left[t_{k}^{l, 0}, t_{k+1}^{l, 0}\right) \\
& u_{l, 0}^{P}(t)=\frac{1}{K_{l, 0}} \beta^{P} \operatorname{sign}_{\varepsilon^{P}}\left(f_{l}^{P}\left(\tau_{\tilde{k}}^{l, 0}\right)\right), \text { for } t \in\left[\tau_{\tilde{k}}^{l, 0}, \tau_{\tilde{k}+1}^{l, 0}\right)
\end{aligned}
$$

where

$$
\operatorname{sign}_{\mathcal{E}}(x)= \begin{cases}\operatorname{sign}(x), & \text { if }|x| \geq \varepsilon \\ 0, & \text { otherwise }\end{cases}
$$

Remark 2. The execution of control law (13) and (14) for $D G_{l, 0}$ only requires the triggered information of the neighboring $D G_{m, 0} \in \tilde{N}_{l}$. This demonstrates that the continuous information transmission is no longer necessary, which further leads to the communication burdens about self-triggered control (13) and (14) being sharply reduced compared with that about traditional control (5) and (6).

Theorem 1. Assuming that at least one leader DG can obtain the information of rated frequency $\omega^{\text {rated, }}$ the inter-MGs distributed secondary control law (13) and (14) with the self-triggered mechanism (9) and (10) restores the frequency $\omega_{l, 0}(l \in\{1, \ldots, s\})$ of each leader $D G_{l, 0}$ to the constant $\bar{\omega}_{l, 0}$ in finite time $T$, where $\left|\bar{\omega}_{l, 0}-\omega^{\text {rated }}\right|<\frac{\sqrt{s} \varepsilon^{\omega}}{\lambda_{\min }(\tilde{L}+\tilde{D})}$. Moreover, if the droop coefficients satisfy $K_{l, 0} P_{l, 0}^{\max }=K_{m, 0} P_{m, 0}^{\max }$ for all $l$ and $m$, the active power output ratios $\frac{P_{l, 0}(t)}{P_{l, 0}^{\text {max }}}$ of each leader $D G_{l, 0}$ converge to the constant $\frac{\bar{P}_{l, 0}}{P_{l, 0}^{\text {max }}}$ in finite time $T$, where $\left|\frac{\bar{P}_{l, 0}}{P_{l, 0} \max }-\frac{\bar{P}_{m, 0}}{P_{m, 0}}\right|<\frac{\sqrt{s} \varepsilon^{P}}{\lambda_{2}(\tilde{L}) K_{l, 0} P_{l, 0}^{\max }}$ for all $l$ and $m . \lambda_{\min }(\cdot)$ and $\lambda_{2}(\cdot)$ denote the minimal and second-largest eigenvalues, respectively, and the matrix $\tilde{D}=\operatorname{diag}\left\{\tilde{a}_{10}, \ldots, \tilde{a}_{s 0}\right\}$.

Proof. The definition of $u_{l, 0}^{\omega}(t)$ shown in Section 2.2 and Equation (13) imply that, for $t \in\left[t_{k}^{l, 0}, t_{k+1}^{l, 0}\right)$,

$$
\frac{d}{d t} \omega_{l, 0}(t)=\beta^{\omega} \operatorname{sign}_{\varepsilon^{\omega}}\left(f_{l}^{\omega}\left(t_{k}^{l, 0}\right)\right)
$$

Defining the vector $\tilde{\omega}=\left(\omega_{1,0}, \ldots, \omega_{s, 0}\right)^{T}-\omega^{\text {rated }} \mathbf{1}_{s}$ where the $s$-dimensional vector $\mathbf{1}_{s}=(1, \ldots, 1)^{T}$, consider the Lyapunov function

$$
V=\frac{1}{2} \tilde{\omega}^{T}(\tilde{L}+\tilde{D}) \tilde{\omega}
$$

where the matrix $\tilde{L}+\tilde{D}$ is positive definite according to [27] under the assumption of Theorem 1. 
The derivation of Lyapunov function (16) with respect to time $t$ along the solution of closed-loop system (15) for $t \in\left[t_{k}^{l, 0}, t_{k+1}^{l, 0}\right)$ is

$$
\begin{aligned}
\frac{d}{d t} V & =\left(\frac{d}{d t} \tilde{\omega}\right)^{T}(\tilde{L}+\tilde{D}) \tilde{\omega} \\
& =\sum_{l=1}^{s} u_{l, 0}^{\omega}(t)\left(\sum_{m=1}^{s} \tilde{a}_{l m}\left(\omega_{l, 0}(t)-\omega_{m, 0}(t)\right)+\tilde{a}_{l 0}\left(\omega_{l, 0}(t)-\omega^{\text {rated }}\right)\right) \\
& =-\beta^{\omega} \sum_{l=1}^{s} \operatorname{sign}_{\varepsilon^{\omega}}\left(f_{l}^{\omega}\left(t_{k}^{l, 0}\right)\right) f_{l}^{\omega}(t) \\
& =-\beta^{\omega} \sum_{l:\left|f_{l}^{\omega}\left(t_{k}^{l, 0}\right)\right| \geq \varepsilon^{\omega}} \operatorname{sign}\left(f_{l}^{\omega}\left(t_{k}^{l, 0}\right)\right) f_{l}^{\omega}(t)
\end{aligned}
$$

Since $\left|u_{l, 0}^{\omega}(t)\right| \leq \beta^{\omega}$ according to Formula (13), it gets

$$
\left|\omega_{l, 0}(t)-\omega_{l, 0}\left(t_{k}^{l, 0}\right)\right|=\left|\int_{t_{k}^{l, 0}}^{t} u_{l, 0}^{\omega}(\tau) d \tau\right| \leq \int_{t_{k}^{l, 0}}^{t}\left|u_{l, 0}^{\omega}(\tau)\right| d \tau \leq \beta^{\omega}\left(t-t_{k}^{l, 0}\right) .
$$

This implies

$$
\begin{aligned}
\omega_{m, 0}(t)-\omega_{l, 0}(t) & \geq \omega_{m, 0}\left(t_{k}^{l, 0}\right)-\omega_{l, 0}\left(t_{k}^{l, 0}\right)-2 \beta^{\omega}\left(t-t_{k}^{l, 0}\right) \\
\omega^{\text {rated }}-\omega_{l, 0}(t) & \geq \omega^{\text {rated }}-\omega_{l, 0}\left(t_{k}^{l, 0}\right)-\beta^{\omega}\left(t-t_{k}^{l, 0}\right)
\end{aligned}
$$

and

$$
\begin{aligned}
\omega_{m, 0}(t)-\omega_{l, 0}(t) & \leq \omega_{m, 0}\left(t_{k}^{l, 0}\right)-\omega_{l, 0}\left(t_{k}^{l, 0}\right)+2 \beta^{\omega}\left(t-t_{k}^{l, 0}\right) \\
\omega^{\text {rated }}-\omega_{l, 0}(t) & \leq \omega^{\text {rated }}-\omega_{l, 0}\left(t_{k}^{l, 0}\right)+\beta^{\omega}\left(t-t_{k}^{l, 0}\right)
\end{aligned}
$$

For $t \in\left[t_{k}^{l, 0}, t_{k+1}^{l, 0}\right)$, Formulas (19)-(22) lead to

$$
\begin{aligned}
& f_{l}^{\omega}(t) \geq f_{l}^{\omega}\left(t_{k}^{l, 0}\right)-\beta^{\omega}\left(2\left|\tilde{N}_{l}\right|+\tilde{a}_{l 0}\right)\left(t-t_{k}^{l, 0}\right) \\
& f_{l}^{\omega}(t) \leq f_{l}^{\omega}\left(t_{k}^{l, 0}\right)+\beta^{\omega}\left(2\left|\tilde{N}_{l}\right|+\tilde{a}_{l 0}\right)\left(t-t_{k}^{l, 0}\right)
\end{aligned}
$$

Combining Formulas (9), (23) and (24) yields that, if $f_{l}^{\omega}\left(t_{k}^{l, 0}\right) \geq \varepsilon^{\omega}$, we have

$$
f_{l}^{\omega}(t) \geq f_{l}^{\omega}\left(t_{k}^{l, 0}\right)-\beta^{\omega}\left(2\left|\tilde{N}_{l}\right|+\tilde{a}_{l 0}\right)\left(t_{k+1}^{l, 0}-t_{k}^{l, 0}\right)=\frac{1}{2} f_{l}^{\omega}\left(t_{k}^{l, 0}\right)
$$

and if $f_{l}^{\omega}\left(t_{k}^{l, 0}\right) \leq-\varepsilon^{\omega}$, we have

$$
f_{l}^{\omega}(t) \leq f_{l}^{\omega}\left(t_{k}^{l, 0}\right)+\beta^{\omega}\left(2\left|\tilde{N}_{l}\right|+\tilde{a}_{l 0}\right)\left(t_{k+1}^{l, 0}-t_{k}^{l, 0}\right)=\frac{1}{2} f_{l}^{\omega}\left(t_{k}^{l, 0}\right)
$$

Formulas (25) and (26) demonstrate that $\operatorname{sign}\left(f_{l}^{\omega}\left(t_{k}^{l, 0}\right)\right)=\operatorname{sign}\left(f_{l}^{\omega}(t)\right)$ if $\left|f_{l}^{\omega}\left(t_{k}^{l, 0}\right)\right|>\varepsilon^{\omega}$, which further means the following Formula (27) can be obtained according to Equation (17)

$$
\begin{aligned}
\frac{d}{d t} V & =-\beta^{\omega} \sum_{l:\left|f_{l}^{\omega}\left(t_{k}^{l, 0}\right)\right| \geq \varepsilon^{\omega}} \operatorname{sign}\left(f_{l}^{\omega}(t)\right) f_{l}^{\omega}(t)=-\beta^{\omega} \sum_{l:\left|f_{l}^{\omega}\left(t_{k}^{l, 0}\right)\right| \geq \varepsilon^{\omega}}\left|f_{l}^{\omega}(t)\right| \\
& \leq-\beta^{\omega} \sum_{l:\left|f_{l}^{\omega}\left(t_{k}^{l, 0}\right)\right| \geq \varepsilon^{\omega}} \frac{1}{2}\left|f_{l}^{\omega}\left(t_{k}^{l, 0}\right)\right| \leq-\beta^{\omega} \sum_{l:\left|f_{l}^{\omega}\left(t_{k}^{l, 0}\right)\right| \geq \varepsilon^{\omega}} \frac{\varepsilon^{\omega}}{2}
\end{aligned}
$$


where the fact $\left|f_{l}^{\omega}(t)\right| \geq \frac{1}{2}\left|f_{l}^{\omega}\left(t_{k}^{l, 0}\right)\right|$ for $\left|f_{l}^{\omega}\left(t_{k}^{l, 0}\right)\right| \geq \varepsilon^{\omega}$ obtained from Formulas (25) and (26) is used for the above inequation.

Formula (27) implies that there exists a fixed finite time instant $T_{1}$ such that

$$
\left\{l:\left|f_{l}^{\omega}\left(t_{k}^{l, 0}\right)\right| \geq \varepsilon^{\omega}\right\}=\varnothing, \quad \text { for } t_{k}^{l, 0} \in\left[T_{1},+\infty\right)
$$

Otherwise, there would be an infinite number of time intervals whose length is lower bounded by the positive constant $\min _{l} \frac{\varepsilon^{\omega}}{2 \beta^{\omega}\left(2\left|\tilde{N}_{l}\right|+\tilde{a}_{l 0}\right)}$ and on which $\frac{d}{d t} V \leq-\frac{1}{2} \beta^{\omega} \varepsilon^{\omega}$, and this contradicts the positive definiteness of function $V$. Formula (28) shows $\left|f_{l}^{\omega}\left(t_{k}^{l, 0}\right)\right|<\varepsilon^{\omega}$ for $t_{k}^{l, 0} \in\left[T_{1},+\infty\right)$ and $l \in\{1, \ldots, s\}$, which means $u_{l, 0}^{\omega}(t)=0$ for $t \in\left[T_{1},+\infty\right)$ according to Formula (13). This further demonstrates

$$
\left|f_{l}^{\omega}(t)\right|<\varepsilon^{\omega}, \quad \text { for } t \in\left[T_{1},+\infty\right) \text { and } \forall l \in\{1, \ldots, s\}
$$

By the help of Formulas (11) and (29), we have $\|(\tilde{L}+\tilde{D}) \tilde{\omega}(t)\|_{2}^{2}<s\left(\varepsilon^{\omega}\right)^{2}$ for $t>T_{1}$ where $\|\cdot\|_{2}$ denotes the $\mathcal{L}_{2}$-norm of vector, and it further means $\|\tilde{\omega}(t)\|_{2}^{2}<\frac{s\left(\varepsilon^{\omega}\right)^{2}}{\lambda_{\text {min }}^{2}(\tilde{L}+\tilde{D})}$ for $t>T_{1}$. As a consequence, it gets that $\lim _{t \rightarrow T_{1}} \omega_{l, 0}(t)=\bar{\omega}_{l, 0}$, where $\left|\bar{\omega}_{l, 0}-\omega^{\text {rated }}\right|<\frac{\sqrt{s \varepsilon} \omega^{\omega n}}{\lambda_{\min }(\tilde{L}+\tilde{D})}$ for $l=1, \ldots, s$.

By a similar analysis, we can get that $\lim _{t \rightarrow T_{2}} P_{l, 0}(t)=\bar{P}_{l, 0}$, where $\left|K_{l, 0} \bar{P}_{l, 0}-K_{m, 0} \bar{P}_{m, 0}\right|<\frac{\sqrt{s \varepsilon} P}{\lambda_{2}(\tilde{L})}$ for all $l$ and $m$ with the fixed finite time instant $T_{2}$. It further leads to the fact that $\left|\frac{\bar{P}_{l, 0}}{P_{l, 0}^{m a x}}-\frac{\bar{P}_{m, 0}}{P_{m, 0}}\right|<$ $\frac{\sqrt{s} \varepsilon^{P}}{\lambda_{2}(\tilde{L}) K_{l, 0} \max }$ since the droop coefficients are set as $K_{l, 0} P_{l, 0}^{\max }=K_{m, 0} P_{m, 0}^{\max }$. Setting $T=\max \left\{T_{1}, T_{2}\right\}$ concludes the proof.

Remark 3. Theorem 1 shows that the fluctuation ranges noted in Purposes 1 and 2 satisfy $\bar{\varepsilon}_{\omega}=\frac{\sqrt{s} \varepsilon^{\omega}}{\lambda_{\min }(\tilde{L}+\tilde{D})}$ and $\bar{\varepsilon}_{P}=\frac{\sqrt{s \varepsilon}^{P}}{\lambda_{2}(\tilde{L}) K_{l, 0} P_{l, 0}^{m a x}}$. As mentioned, tuning the parameters $\varepsilon^{\omega}$ and $\varepsilon^{P}$ in the self-triggered mechanism (9) and (10) and the inter-MGs control law (13) and (14) can make these fluctuation ranges acceptable. Decreasing these parameters can reduce the fluctuation ranges to guarantee better control accuracy. However, it also reduces the lower bounds of inter-trigger time intervals $t_{k+1}^{l, 0}-t_{k}^{l, 0}$ and $\tau_{\tilde{k}+1}^{l, 0}-\tau_{\tilde{k}}^{l, 0}$ as illustrated in Remark 1, which may lead to serious communication burdens in the inter-MGs communication network. On the contrary, increasing these parameters could reduce the communication burdens further; however, it enlarges the fluctuation ranges. In the engineering practice, the parameters $\varepsilon^{\omega}$ and $\varepsilon^{P}$ are selected weighting the factors of the fluctuation tolerant level and the communication resource limitation.

Substituting Formulas (13) and (14) into Equation (2) yields the regulation of frequency nominal set-point about each leader $D G_{l, 0}$ for $t \in\left[t_{k}^{l, 0}, t_{k+1}^{l, 0}\right) \cap\left[\tau_{\tilde{k}}^{l, 0}, \tau_{\tilde{k}+1}^{l, 0}\right)$ as

$$
\omega_{l, 0}^{n o m}(t)=\int\left(\beta^{\omega} \operatorname{sign}_{\varepsilon^{\omega}}\left(f_{l}^{\omega}\left(t_{k}^{l, 0}\right)\right)+\beta^{P} \operatorname{sign}_{\mathcal{E}^{P}}\left(f_{l}^{P}\left(\tau_{\tilde{k}^{l, 0}}^{l, 0}\right)\right) d t\right.
$$

\subsection{Event-Triggered Mechanism Based Intra-MG Distributed Secondary Control}

The intra-MG distributed secondary control aims to drive the frequency $\omega_{l, i}$ and the active power output ratio $\frac{P_{l, i}(t)}{P_{l, i}^{\max }}$ of each follower $D G_{l, i}$ in $M G_{l}$ to the values $\bar{\omega}_{l, 0}$ and $\frac{\bar{P}_{l, 0}}{P_{l, 0} \text { max }}$ of the leader $D G_{l, 0}$, respectively. In order to reduce the communication burdens, the leader $D G_{l, 0}$ transmits its own frequency and active power output ratio information to some follower DGs in $M G_{l}$ periodically with the sampling period $\Delta T$. Moreover, the event-triggered mechanism is configured at each follower DG to determine the triggering 
time instants of information transmission among the follower DGs through the intra-MG communication network. Considering the follower DGs in $M G_{l}$, define the measurement errors of $D G_{l, i}$ as

$$
\begin{aligned}
& e_{l, i}^{\omega}(t)=\omega_{l, i}\left(t_{k}^{l, i}\right)-\omega_{l, i}(t), \text { for } t \in\left[t_{k}^{l, i}, t_{k+1}^{l, i}\right) \\
& e_{l, i}^{P}(t)=K_{l, i}\left(P_{l, i}\left(\tau_{\tilde{k}}^{l, i}\right)-P_{l, i}(t)\right), \text { for } t \in\left[\tau_{\tilde{k}}^{l, i}, \tau_{\tilde{k}+1}^{l, i}\right)
\end{aligned}
$$

Event-triggered mechanism: For the follower $D G_{l, i}$ in $M G_{l}$, denoting the last triggering time for frequency $\omega_{l, i}$ [active power output $P_{l, i}$ resp.] as $t_{k}^{l, i}\left[\tau_{\tilde{k}}^{l, i}\right.$ resp.], $D G_{l, i}$ transmits its own information $\omega_{l, i}\left(t_{k}^{l, i}\right)\left[P_{l, i}\left(\tau_{\tilde{k}}^{l, i}\right)\right.$ resp.] to its neighbors $D G_{l, j} \in N_{i}^{l}$ in intra-MG communication network $\mathcal{G}^{l}$ at time $t_{k}^{l, i}$ [ $\tau_{\tilde{k}}^{l, i}$ resp.]. Moreover, the next triggering time is calculated at $D G_{l, i}$ according to Formulas (33) and (34), and the above action will be repeated at $t_{k+1}^{l, i}\left[\tau_{\tilde{k}+1}^{l, i}\right.$ resp.]:

$$
\begin{aligned}
& t_{k+1}^{l, i}=\inf _{t>t_{k}^{l, i}}\left\{t \mid\left(\left|N_{i}^{l}\right|+\frac{a_{i 0}^{l}}{2}\right)\left(e_{l, i}^{\omega}(t)\right)^{2}>\sigma_{l, i}^{\omega}\left(\frac{1}{4} \sum_{j=1}^{n^{l}} a_{i j}^{l}\left(\omega_{l, i}\left(t_{k}^{l, i}\right)-\omega_{l, j}\left(t_{k^{\prime}(t)}^{l, j}\right)\right)^{2}\right.\right. \\
& \left.\left.+\frac{1}{2} a_{i 0}^{l}\left(\omega_{l, i}\left(t_{k}^{l, i}\right)-\omega_{l, 0}\left(k^{\prime}(t) \Delta T\right)\right)^{2}\right)+\phi_{l, i}^{\omega}(t)\right\} \\
& \tau_{\tilde{k}+1}^{l, i}=\inf _{t>\tau_{\tilde{k}}^{l, j}}\left\{t \mid\left(\left|N_{i}^{l}\right|+\frac{a_{i 0}^{l}}{2}\right)\left(e_{l, i}^{P}(t)\right)^{2}>\sigma_{l, i}^{P}\left(\frac{1}{4} \sum_{j=1}^{n^{l}} a_{i j}^{l}\left(K_{l, i} P_{l, i}\left(\tau_{\tilde{k}}^{l, i}\right)-K_{l, j} P_{l, j}\left(\tau_{\tilde{k}^{\prime}(t)}^{l, j}\right)\right)^{2}\right.\right. \\
& \left.\left.+\frac{1}{2} a_{i 0}^{l}\left(K_{l, i} P_{l, i}\left(\tau_{\tilde{k}}^{l, i}\right)-K_{l, 0} P_{l, 0}\left(\tilde{k}^{\prime}(t) \Delta T\right)\right)^{2}\right)+\phi_{l, i}^{P}(t)\right\}
\end{aligned}
$$

and the dynamic behavior of internal dynamic variable $\phi_{l, i}^{\omega}(t)$ and $\phi_{l, i}^{P}(t)$ in Formulas (33) and (34) satisfies

$$
\begin{aligned}
& \frac{d}{d t} \phi_{l, i}^{\omega}(t)=-\alpha_{l, i}^{\omega} \phi_{l, i}^{\omega}(t)+\gamma_{l, i}^{\omega}( \sigma_{l, i}^{\omega}\left(\frac{1}{4} \sum_{j=1}^{n^{l}} a_{i j}^{l}\left(\omega_{l, i}\left(t_{k}^{l, i}\right)-\omega_{l, j}\left(t_{k^{\prime}(t)}^{l, j}\right)\right)^{2}\right. \\
&\left.\left.+\frac{1}{2} a_{i 0}^{l}\left(\omega_{l, i}\left(t_{k}^{l, i}\right)-\omega_{l, 0}\left(k^{\prime}(t) \Delta T\right)\right)^{2}\right)-\left(\left|N_{i}^{l}\right|+\frac{a_{i 0}^{l}}{2}\right)\left(e_{l, i}^{\omega}(t)\right)^{2}\right) \\
& \frac{d}{d t} \phi_{l, i}^{P}(t)=-\alpha_{l, i}^{P} \phi_{l, i}^{P}(t)+\gamma_{l, i}^{P}\left(\sigma _ { l , i } ^ { P } \left(\frac{1}{4} \sum_{j=1}^{n^{l}} a_{i j}^{l}\left(K_{l, i} P_{l, i}\left(\tau_{\tilde{k}}^{l, i}\right)-K_{l, j} P_{l, j}\left(\tau_{\tilde{k}^{\prime}(t)}^{l, j}\right)\right)^{2}\right.\right. \\
&\left.\left.+\frac{1}{2} a_{i 0}^{l}\left(K_{l, i} P_{l, i}\left(\tau_{\tilde{k}^{l, i}}^{l, i}\right)-K_{l, 0} P_{l, 0}\left(\tilde{k}^{\prime}(t) \Delta T\right)\right)^{2}\right)-\left(\left|N_{i}^{l}\right|+\frac{a_{i 0}^{l}}{2}\right)\left(e_{l, i}^{P}(t)\right)^{2}\right)
\end{aligned}
$$

with the initial values $\phi_{l, i}^{\omega}(0)>0$ and $\phi_{l, i}^{P}(0)>0$, where the constants $\alpha_{l, i}^{\omega}>1, \alpha_{l, i}^{P}>1$, $\gamma_{l, i}^{\omega}>0, \gamma_{l, i}^{P}>0,0<\sigma_{l, i}^{\omega}<\frac{1}{1+\gamma_{l, i}^{\omega}}$ and $0<\sigma_{l, i}^{P}<\frac{1}{1+\gamma_{l, i}^{P}}, \omega_{l, j}\left(t_{k^{\prime}(t)}^{l, j}\right)\left[P_{l, j}\left(\tau_{\tilde{k}^{\prime}(t)}^{l, j}\right)\right.$ resp. $]$ denotes the latest triggered information about frequency [active power output.] of $D G_{l, j}$ before time $t$, and $\omega_{l, 0}\left(k^{\prime}(t) \Delta T\right)\left[P_{l, 0}\left(\tilde{k}^{\prime}(t) \Delta T\right)\right.$ resp.] represents the latest periodic sampling information about frequency [active power output.] of the leader $D G_{l, 0}$ before time $t$.

Remark 4. The implementation of event-triggered mechanism (33) and (34) for the follower $D G_{l, i}$ only requires the triggered information of the neighboring DGs in $N_{i}^{l}$ in the intra-MG communication network. This demonstrates that the event-triggered mechanism is compatible with the distributed architecture.

Lemma 1. The internal dynamic variable $\phi_{l, i}^{\omega}(t)$ and $\phi_{l, i}^{P}(t)$ in the mechanism (33) and (34) satisfy

$$
\begin{aligned}
& \phi_{l, i}^{\omega}(t) \geq \phi_{l, i}^{\omega}(0) e^{-\left(\alpha_{l, i}^{\omega}+\gamma_{l, i}^{\omega}\right) t} \\
& \phi_{l, i}^{P}(t) \geq \phi_{l, i}^{P}(0) e^{-\left(\alpha_{l, i}^{P}+\gamma_{l, i}^{P}\right) t}
\end{aligned}
$$


Proof. The event-triggered mechanism (33) demonstrates that, for $t \in\left[t_{k}^{l, i}, t_{k+1}^{l, i}\right)$,

$$
\left(\left|N_{i}^{l}\right|+\frac{a_{i 0}^{l}}{2}\right)\left(e_{l, i}^{\omega}(t)\right)^{2} \leq \sigma_{l, i}^{\omega}\left(\frac{1}{4} \sum_{j=1}^{n^{l}} a_{i j}^{l}\left(\omega_{l, i}\left(t_{k}^{l, i}\right)-\omega_{l, j}\left(t_{k^{\prime}(t)}^{l, j}\right)\right)^{2}+\frac{1}{2} a_{i 0}^{l}\left(\omega_{l, i}\left(t_{k}^{l, i}\right)-\omega_{l, 0}\left(k^{\prime}(t) \Delta T\right)\right)^{2}\right)+\phi_{l, i}^{\omega}(t) .
$$

Substituting Inequation (39) into Formula (35) yields $\frac{d}{d t} \phi_{l, i}^{\omega}(t) \geq-\left(\alpha_{l, i}^{\omega}+\gamma_{l, i}^{\omega}\right) \phi_{l, i}^{\omega}(t)$, and then Inequation (37) can be obtained according to the comparison lemma. As mentioned, Inequation (38) can be obtained by similar analysis.

Based on the event-triggered mechanism, the intra-MG distributed secondary control law is given as follows:

$$
\begin{array}{r}
u_{l, i}^{\omega}(t)=\beta_{l}^{\omega}\left(\sum_{j=1}^{n^{l}} a_{i j}^{l}\left(\omega_{l, j}\left(t_{k^{\prime}(t)}^{l, j}\right)-\omega_{l, i}\left(t_{k}^{l, i}\right)\right)+a_{i 0}^{l}\left(\omega_{l, 0}\left(k^{\prime}(t) \Delta T\right)-\omega_{l, i}\left(t_{k}^{l, i}\right)\right)\right), \text { for } t \in\left[t_{k}^{l, i}, t_{k+1}^{l, i}\right) \\
u_{l, i}^{P}(t)=\frac{\beta_{l}^{P}}{K_{l, i}}\left(\sum_{j=1}^{n^{l}} a_{i j}^{l}\left(K_{l, j} P_{l, j}\left(\tau_{\tilde{k}^{\prime}(t)}^{l, j}\right)-K_{l, i} P_{l, i}\left(\tau_{\tilde{k}}^{l, i}\right)\right)+a_{i 0}^{l}\left(K_{l, 0} P_{l, 0}\left(\tilde{k}^{\prime}(t) \Delta T\right)-K_{l, i} P_{l, i}\left(\tau_{\tilde{k}}^{l, i}\right)\right)\right), \\
\text { for } t \in\left[\tau_{\tilde{k}}^{l, i}, \tau_{\tilde{k}+1}^{l, i}\right)
\end{array}
$$

where $\beta_{l}^{\omega}$ and $\beta_{l}^{P}$ are positive constants.

Remark 5. Compared with the traditional control law (7) and (8) based on the continuous information transmission, the execution of event-triggered intra-MG control law (40) and (41) for the follower $D G_{l, i}$ only requires the discrete triggered information of its neighbors in $N_{i}^{l}$. This implies that less information is needed to be transmitted, and the communication burdens can be reduced.

Without loss of generality, the following theory is given only for $M G_{l}$. The result of other MG can be obtained similarly.

Theorem 2. Assuming that at least one follower $D G$ in $M G_{l}$ can obtain the information of leader $D G_{l, 0}$, the intra-MG distributed secondary controls (40) and (41) with the event-triggered mechanism (33) and (34) restores the frequency $\omega_{l, i}\left(i \in\left\{1, \ldots, n^{l}\right\}\right)$ of each follower $D G_{l, i}$ to $\bar{\omega}_{l, 0}$. Moreover, if the droop coefficients satisfy $K_{l, i} P_{l, i}^{\max }=K_{l, j} P_{l, j}^{\max }$ for all $i$ and $j$, the active power output ratios $\frac{P_{l, i}(t)}{P_{l, i}^{\text {max }}}$ converge to $\frac{\bar{P}_{l, 0}}{P_{l, 0}^{\max }}$. The constants $\bar{\omega}_{l, 0}$ and $\bar{P}_{l, 0}$ are given in Theorem 1 .

Proof. Since $\lim _{t \rightarrow T} \omega_{l, 0}(t)=\bar{\omega}_{l, 0}$ as proved in Theorem 1, we get that $\omega_{l, 0}(t) \equiv \bar{\omega}_{l, 0}$ for $t \in[T,+\infty)$. The following proof only considers the dynamical behavior in the time interval $[T,+\infty)$ where the variable $\omega_{l, 0}(t)$ can be replaced by the constant $\bar{\omega}_{l, 0}$. The definition of $u_{l, i}^{\omega}(t)$ shown in Section 2.2 and Equation (40) imply that, for $t \in\left[t_{k}^{l, i}, t_{k+1}^{l, i}\right)$, we have

$$
u_{l, i}^{\omega}(t)=\frac{d}{d t} \omega_{l, i}(t)=\beta_{l}^{\omega}\left(\sum_{j=1}^{n^{l}} a_{i j}^{l}\left(\omega_{l, j}\left(t_{k^{\prime}(t)}^{l, j}\right)-\omega_{l, i}\left(t_{k}^{l, i}\right)\right)+a_{i 0}^{l}\left(\bar{\omega}_{l, 0}-\omega_{l, i}\left(t_{k}^{l, i}\right)\right)\right)
$$

Defining the matrix $D^{l}=\operatorname{diag}\left\{a_{10}^{l}, \ldots, a_{n^{l} 0}^{l}\right\}$ and the vector $\tilde{\omega}_{l}=\left\{\tilde{\omega}_{l, 1}, \tilde{\omega}_{l, 2}, \ldots, \tilde{\omega}_{l, n^{l}}\right\}^{T} \in R^{n^{l}}$ where $\tilde{\omega}_{l, i}=\omega_{l, i}-\bar{\omega}_{l, 0}$ for $i=1, \ldots, n^{l}$, consider the Lyapunov function

$$
V^{l}=\frac{1}{2 \beta_{l}^{\omega}}\left(\tilde{\omega}_{l}(t)\right)^{T} \tilde{\omega}_{l}(t)+\sum_{i=1}^{n^{l}} \phi_{l, i}^{\omega}(t)
$$


The derivation of Lyapunov function (43) with respect to time $t$ along the solution of closed-loop system (42) for $t \in\left[t_{k}^{l, i}, t_{k+1}^{l, i}\right)$ is

$$
\frac{d}{d t} V^{l}=\sum_{i=1}^{n^{l}}\left(\frac{1}{\beta_{l}^{\omega}} \tilde{\omega}_{l, i}(t) u_{l, i}^{\omega}(t)+\frac{d}{d t} \phi_{l, i}^{\omega}(t)\right)=\frac{1}{\beta_{i}^{\omega}}\left(\sum_{i=1}^{n^{l}} \tilde{\omega}_{l, i}\left(t_{k}^{l, i}\right) u_{l, i}^{\omega}(t)-\sum_{i=1}^{n^{l}} e_{l, i}^{\omega}(t) u_{l, i}^{\omega}(t)\right)+\sum_{i=1}^{n^{l}} \frac{d}{d t} \phi_{l, i}^{\omega}(t)
$$

Based on Equation (42), we have

$$
\begin{aligned}
& \sum_{i=1}^{n^{l}} \tilde{\omega}_{l, i}\left(t_{k}^{l, i}\right) u_{l, i}^{\omega}(t) \\
= & \beta_{l}^{\omega} \sum_{i=1}^{n^{l}} \tilde{\omega}_{l, i}\left(t_{k}^{l, i}\right)\left(\sum_{j=1}^{n^{l}} a_{i j}^{l}\left(\tilde{\omega}_{l, j}\left(t_{k^{\prime}(t)}^{l, j}\right)-\tilde{\omega}_{l, i}\left(t_{k}^{l, i}\right)\right)-a_{i 0}^{l} \tilde{\omega}_{l, i}\left(t_{k}^{l, i}\right)\right) \\
= & -\frac{\beta_{l}^{\omega}}{2} \sum_{i=1}^{n^{l}} \sum_{j=1}^{n^{l}} a_{i j}^{l}\left(\tilde{\omega}_{l, j}\left(t_{k^{\prime}(t)}^{l, j}\right)-\tilde{\omega}_{l, i}\left(t_{k}^{l, i}\right)\right)^{2}-\beta_{l}^{\omega} \sum_{i=1}^{n^{l}} a_{i 0}^{l}\left(\tilde{\omega}_{l, i}\left(t_{k}^{l, i}\right)\right)^{2}
\end{aligned}
$$

where $\sum_{i=1}^{n^{l}} \sum_{j=1}^{n^{l}} a_{i j}^{l}\left(\tilde{\omega}_{l, j}\left(t_{k^{\prime}(t)}^{l, j}\right)\right)^{2}=\sum_{i=1}^{n^{l}} \sum_{j=1}^{n^{l}} a_{i j}^{l}\left(\tilde{\omega}_{l, i}\left(t_{k}^{l, i}\right)\right)^{2}$ is used here since the graph $\mathcal{G}^{l}$ is undirected and connected.

By the help of Young's inequality, we get

$$
\begin{aligned}
& -\sum_{i=1}^{n^{l}} e_{l, i}^{\omega}(t) u_{l, i}^{\omega}(t) \\
= & -\beta_{l}^{\omega} \sum_{i=1}^{n^{l}} e_{l, i}^{\omega}(t)\left(\sum_{j=1}^{n^{l}} a_{i j}^{l}\left(\tilde{\omega}_{l, j}\left(t_{k^{\prime}(t)}^{l, j}\right)-\tilde{\omega}_{l, i}\left(t_{k}^{l, i}\right)\right)-a_{i 0}^{l} \tilde{\omega}_{l, i}\left(t_{k}^{l, i}\right)\right) \\
\leq & \beta_{l}^{\omega} \sum_{i=1}^{n^{l}}\left|N_{i}^{l}\right|\left(e_{l, i}^{\omega}(t)\right)^{2}+\frac{\beta_{l}^{\omega}}{4} \sum_{i=1}^{n^{l}} \sum_{j=1}^{n^{l}} a_{i j}^{l}\left(\tilde{\omega}_{l, j}\left(t_{k^{\prime}(t)}^{l, j}\right)-\tilde{\omega}_{l, i}\left(t_{k}^{l, i}\right)\right)^{2}+\beta_{l}^{\omega} \sum_{i=1}^{n^{l}} a_{i 0}^{l} e_{l, i}^{\omega}(t) \tilde{\omega}_{l, i}\left(t_{k}^{l, i}\right) \\
\leq & \beta_{l}^{\omega} \sum_{i=1}^{n^{l}}\left(\left|N_{i}^{l}\right|+\frac{a_{i 0}^{l}}{2}\right)\left(e_{l, i}^{\omega}(t)\right)^{2}+\beta_{l}^{\omega} \sum_{i=1}^{n^{l}} \sum_{j=1}^{n^{l}} \frac{a_{i j}^{l}}{4}\left(\tilde{\omega}_{l, j}\left(t_{k^{\prime}(t)}^{l, j}\right)-\tilde{\omega}_{l, i}\left(t_{k}^{l, i}\right)\right)^{2}+\beta_{l}^{\omega} \sum_{i=1}^{n^{l}} \frac{a_{i 0}^{l}}{2}\left(\tilde{\omega}_{l, i}\left(t_{k}^{l, i}\right)\right)^{2}
\end{aligned}
$$

Combining Formulas (44)-(46) yields

$$
\frac{d}{d t} V^{l} \leq \sum_{i=1}^{n^{l}}\left(\left(\left|N_{i}^{l}\right|+\frac{a_{i 0}^{l}}{2}\right)\left(e_{l, i}^{\omega}(t)\right)^{2}-\sum_{j=1}^{n^{l}} \frac{a_{i j}^{l}}{4}\left(\tilde{\omega}_{l, j}\left(t_{k^{\prime}(t)}^{l, j}\right)-\tilde{\omega}_{l, i}\left(t_{k}^{l, i}\right)\right)^{2}-\frac{a_{i 0}^{l}}{2}\left(\tilde{\omega}_{l, i}\left(t_{k}^{l, i}\right)\right)^{2}+\frac{d}{d t} \phi_{l, i}^{\omega}(t)\right)
$$

According to the event-triggered mechanism (33), we can obtain for $t \in\left[t_{k}^{l, i}, t_{k+1}^{l, i}\right)$,

$$
\left(\left|N_{i}^{l}\right|+\frac{a_{i 0}^{l}}{2}\right)\left(e_{l, i}^{\omega}(t)\right)^{2} \leq \sigma_{l, i}^{\omega}\left(\sum_{j=1}^{n^{l}} \frac{a_{i j}^{l}}{4}\left(\tilde{\omega}_{l, i}\left(t_{k}^{l, i}\right)-\tilde{\omega}_{l, j}\left(t_{k^{\prime}(t)}^{l, j}\right)\right)^{2}+\frac{a_{i 0}^{l}}{2}\left(\tilde{\omega}_{l, i}\left(t_{k}^{l, i}\right)\right)^{2}\right)+\phi_{l, i}^{\omega}(t)
$$

Substituting Formulas (35) and (48) into (47) gets

$$
\begin{aligned}
\frac{d}{d t} V^{l} \leq & \sum_{i=1}^{n^{l}}\left(\left(\sigma_{l, i}^{\omega}+\gamma_{l, i}^{\omega} \sigma_{l, i}^{\omega}-1\right)\left(\sum_{j=1}^{n^{l}} \frac{a_{i j}^{l}}{4}\left(\tilde{\omega}_{l, i}\left(t_{k}^{l, i}\right)-\tilde{\omega}_{l, j}\left(t_{k^{\prime}(t)}^{l, j}\right)\right)^{2}+\frac{a_{i 0}^{l}}{2}\left(\tilde{\omega}_{l, i}\left(t_{k}^{l, i}\right)\right)^{2}\right)\right. \\
& \left.+\left(1-\alpha_{l, i}^{\omega}\right) \phi_{l, i}^{\omega}(t)-\gamma_{l, i}^{\omega}\left(\left|N_{i}^{l}\right|+\frac{a_{i 0}^{l}}{2}\right)\left(e_{l, i}^{\omega}(t)\right)^{2}\right) \\
\leq & 0
\end{aligned}
$$


where the Lemma 1 is used. Formula (49) implies that the solution $\omega_{l, i}(t)$ of the closed-loop system (42) converges to $\bar{\omega}_{l, 0}$ for $i \in\left\{1, \ldots, n^{l}\right\}$ by applying LaSalle's invariance principle.

Moreover, it obtains that $K_{l, i} P_{l, i}(t)$ converges to $K_{l, i} \bar{P}_{l, 0}$ for $i \in\left\{1, \ldots, n^{l}\right\}$ by similar analysis. This together with the fact that $K_{l, i} P_{l, i}^{\max }=K_{l, j} P_{l, j}^{\max }(\forall i, j)$ yields that $\frac{P_{l, i}(t)}{P_{l, i}^{\text {max }}}$ converges to $\frac{\bar{P}_{l, 0}}{P_{l, 0}^{\text {max }}}$. This concludes the proof.

As mentioned, Zeno behavior is impractical for the physical islanded MGs cluster since it leads DGs to trigger an infinite number of events at an accumulation time. Different from the self-triggered mechanisms (9) and (10), the lower bound of inter-trigger time interval of the event-triggered mechanism (33) and (34) is problematic and not obvious. This leads the following theorem to analyze the Zeno behavior of the event-triggered mechanism.

Theorem 3. Zeno behavior can be avoided for the event-triggered mechanism (33) and (34).

Proof. As mentioned, $\left|\frac{d}{d t} \omega_{l, i}(t)\right| \leq M$ with some constant $M$ since the asymptotically stability of the continuous variable $\omega_{l, i}(t)$ is obtained in Theorem 2. Assume that there exists Zeno behavior for the event-triggered mechanism (33), and then it obtains that $\lim _{k \rightarrow+\infty} t_{k}^{l, i}=T_{z}$ for the accumulation time $T_{z}$. By the definition of limitation, it obtains that, for the given positive constant $\varepsilon_{z}=\frac{\sqrt{\phi_{l, i}^{\omega}(0)}}{2 M \sqrt{\left|N_{i}^{l}\right|+\frac{a_{i 0}^{l}}{2}}} e^{-\frac{1}{2}\left(\alpha_{l, i}^{\omega}+\gamma_{l, i}^{\omega}\right) T_{z}}$, there exists a positive integer $N\left(\varepsilon_{z}\right)$, such that

$$
t_{k}^{l, i} \in\left[T_{z}-\varepsilon_{z}, T_{z}\right), \quad \forall k \geq N\left(\varepsilon_{z}\right)
$$

Considering the following formula for $t \in\left(t_{k}^{l, i}, T_{z}\right)$

$$
M\left(t-t_{k}^{l, i}\right) \leq \frac{\sqrt{\phi_{l, i}^{\omega}(0)}}{\sqrt{\left|N_{i}^{l}\right|+\frac{a_{i 0}^{l}}{2}}} e^{-\frac{1}{2}\left(\alpha_{l, i}^{\omega}+\gamma_{l, i}^{\omega}\right) T_{z}}
$$

then we have

$$
\begin{aligned}
\left|e_{l, i}^{\omega}(t)\right| & =\left|\omega_{l, i}\left(t_{k}^{l, i}\right)-\omega_{l, i}(t)\right|=\left|\int_{t_{k}^{l, j}}^{t} \frac{d}{d s} \omega_{l, i}(s)\right| \leq M\left(t-t_{k}^{l, i}\right) \leq \frac{\sqrt{\phi_{l, i}^{\omega}(0)}}{\sqrt{\left|N_{i}^{l}\right|+\frac{a_{i 0}^{l}}{2}}} e^{-\frac{1}{2}\left(\alpha_{l, i}^{\omega}+\gamma_{l, i}^{\omega}\right) T_{z}} \\
& \leq \frac{\sqrt{\phi_{l, i}^{\omega}(0)}}{\sqrt{\left|N_{i}^{l}\right|+\frac{a_{i 0}^{l}}{2}}} e^{-\frac{1}{2}\left(\alpha_{l, i}^{\omega}+\gamma_{l, i}^{\omega}\right) t}=\frac{\sqrt{\phi_{l, i}^{\omega}(t)}}{\sqrt{\left|N_{i}^{l}\right|+\frac{a_{i 0}^{l}}{2}}}
\end{aligned}
$$

where Inequation (37) is used. Formula (52) means that

$$
\left(e_{l, i}^{\omega}(t)\right)^{2} \leq \frac{1}{\left|N_{i}^{l}\right|+\frac{a_{i 0}^{l}}{2}}\left(\phi_{l, i}^{\omega}(t)+\sigma_{l, i}^{\omega}\left(\frac{1}{4} \sum_{j=1}^{n^{l}} a_{i j}^{l}\left(\omega_{l, i}\left(t_{k}^{l, i}\right)-\omega_{l, j}\left(t_{k^{\prime}(t)}^{l, j}\right)\right)^{2}+\frac{1}{2} a_{i 0}^{l}\left(\omega_{l, i}\left(t_{k}^{l, i}\right)-\bar{\omega}_{l, 0}\right)^{2}\right)\right)
$$

This demonstrates that Formula (51) is the sufficient condition of Formula (53), and it further implies

$$
\left(e_{l, i}^{\omega}(t)\right)^{2}>\frac{1}{\left|N_{i}^{l}\right|+\frac{a_{i 0}^{l}}{2}}\left(\phi_{l, i}^{\omega}(t)+\sigma_{l, i}^{\omega}\left(\frac{1}{4} \sum_{j=1}^{n^{l}} a_{i j}^{l}\left(\omega_{l, i}\left(t_{k}^{l, i}\right)-\omega_{l, j}\left(t_{k^{\prime}(t)}^{l, j}\right)\right)^{2}+\frac{1}{2} a_{i 0}^{l}\left(\omega_{l, i}\left(t_{k}^{l, i}\right)-\bar{\omega}_{l, 0}\right)^{2}\right)\right)
$$


is the sufficient condition of

$$
M\left(t-t_{k}^{l, i}\right)>\frac{\sqrt{\phi_{l, i}^{\omega}(0)}}{\sqrt{\left|N_{i}^{l}\right|+\frac{a_{i 0}^{l}}{2}}} e^{-\frac{1}{2}\left(\alpha_{l, i}^{\omega}+\gamma_{l, i}^{\omega}\right) T_{z}}
$$

Letting $k=N\left(\varepsilon_{z}\right)$ and $t=t_{k+1}^{l, i}$, Formula (54) naturally holds based on the event-triggered mechanism (33) since $t_{N\left(\varepsilon_{z}\right)}^{l, i}$ and $t_{N\left(\varepsilon_{z}\right)+1}^{l, i}$ are two neighboring triggering time instants. This further means $t_{N\left(\varepsilon_{z}+1\right)}^{l, i}-t_{N\left(\varepsilon_{z}\right)}^{l, i}>\frac{\sqrt{\phi_{l, i}^{\omega}(0)}}{M \sqrt{\left|N_{i}^{l}\right|+\frac{a_{i 0}^{l}}{2}}} e^{-\frac{1}{2}\left(\alpha_{l, i}^{\omega}+\gamma_{l, i}^{\omega}\right) T_{z}}=2 \varepsilon_{z}$, which is in contradiction with Formula (50). It demonstrates that the existence assumption about Zeno behavior for the event-triggered mechanism (33) is invalid. The result about event-triggered mechanism (34) can be proved by similar analysis.

Substituting control law (40) and (41) into Equation (2) gets the frequency nominal set-point regulation strategy for each follower $D G_{l, i}$ for $t \in\left[t_{k}^{l, i}, t_{k+1}^{l, i}\right) \cap\left[\tau_{\tilde{k}}^{l, i}, \tau_{\tilde{k}+1}^{l, i}\right)$ as

$$
\begin{aligned}
\omega_{l, i}^{n o m}(t)=\int( & \sum_{j=1}^{n^{l}} a_{i j}^{l}\left(\omega_{l, j}\left(t_{k^{\prime}(t)}^{l, j}\right)-\omega_{l, i}\left(t_{k}^{l, i}\right)+K_{l, j} P_{l, j}\left(\tau_{\tilde{k}^{\prime}(t)}^{l, j}\right)-K_{l, i} P_{l, i}\left(\tau_{\tilde{k}}^{l, i}\right)\right) \\
& \left.+a_{i 0}^{l}\left(\omega_{l, 0}\left(k^{\prime}(t) \Delta T\right)-\omega_{l, i}\left(t_{k}^{l, i}\right)+K_{l, 0} P_{l, 0}\left(\tilde{k}^{\prime}(t) \Delta T\right)-K_{l, i} P_{l, i}\left(\tau_{\tilde{k}}^{l, i}\right)\right)\right) d t
\end{aligned}
$$

\subsection{Distributed Hybrid-Triggered Secondary Control for Islanded MGs Cluster}

Combining the self-triggered inter-MGs control and event-triggered intra-MG control yields the hybrid-triggered mechanism based distributed secondary control for the whole islanded MGs cluster.

Theorem 4. Setting the droop coefficient as $K_{l, i} P_{l, i}^{\max }=K$ with some positive constant $K$ for all DGs in the islanded MGs cluster, the inter-MGs control law (30) with the self-triggered mechanism (9) and (10) for the leader DGs and the intra-MG control law (56) with the event-triggered mechanism (33) and (34) for the follower DGs accomplish Purposes 1 and 2 with the fluctuation ranges $\bar{\varepsilon}_{\omega}=\frac{\sqrt{s} \varepsilon^{\omega}}{\lambda_{\min }(\tilde{L}+\tilde{D})}$ and $\bar{\varepsilon}_{P}=\frac{\sqrt{s} \varepsilon^{P}}{\lambda_{2}(\tilde{L}) K}$. Moreover, Zeno behavior can be avoided.

Proof. This theorem can be proved obviously according to Theorems 1-3, and it is omitted here.

\section{Simulation}

This section verifies the effectiveness of theorem by a test system of islanded MGs cluster in Matlab simulation, which contains three MGs with eleven DGs and some loads. The leader DGs in the three MGs are $D G_{1,0}, D G_{2,0}$ and $D G_{3,0}$, and the others are follower DGs. The topology of the test system is shown in Figure 2. The three MGs are physically connected through resistive-inductive lines and the power lines between DGs are series RL branches, and the links in the inter-MGs and intra-MG communication network are given by the different arrow lines as shown by the legend in Figure 2. The rated frequency is set as $\omega^{\text {rated }}=50 \mathrm{~Hz}$, and it is only transmitted to $D G_{1,0}$ in $M G_{1}$. The maximum capacity of the active power output of DGs is given as $P_{1,0}^{\max }=P_{1,1}^{\max }=P_{1,2}^{\max }=P_{1,3}^{\max }=40 \mathrm{~kW}$, $P_{2,0}^{\max }=P_{2,1}^{\max }=P_{2,2}^{\max }=P_{2,3}^{\max }=30 \mathrm{~kW}$ and $P_{3,0}^{\max }=P_{3,1}^{\max }=P_{3,2}^{\max }=60 \mathrm{~kW}$. Each of the load power is $26 \mathrm{~kW}$ at the initial state. Moreover, the droop coefficients are set as $K_{1,0}=K_{1,1}=K_{1,2}=K_{1,3}=$ $3 \times 10^{-5}, K_{2,0}=K_{2,1}=K_{2,2}=K_{2,3}=4 \times 10^{-5}$ and $K_{3,0}=K_{3,1}=K_{3,2}=2 \times 10^{-5}$ in order to satisfy the precondition of Theorem 4 that $K_{l, i} P_{l, i}^{\max }=K$ with some positive constant $K$ for all DGs. The parameters in the self-triggered inter-MGs control law (9)-(14) are selected as $\beta^{\omega}=1, \beta^{P}=0.5$, $\varepsilon^{\omega}=0.2$ and $\varepsilon^{P}=0.1$, and those in the event-triggered intra-MG control law (33)-(36) and (40)-(41) 
are chosen as $\alpha_{l, i}^{\omega}=2, \alpha_{l, i}^{P}=1, \gamma_{l, i}^{\omega}=0.1, \gamma_{l, i}^{P}=0.2, \sigma_{l, i}^{\omega}=0.3, \sigma_{l, i}^{P}=0.4, \beta_{l}^{\omega}=5$ and $\beta_{l}^{P}=7$, which satisfy the parameter requirements in theoretical analysis that $\beta^{\omega}>0, \varepsilon^{\omega}>0, \beta^{P}>0, \varepsilon^{P}>0, \gamma_{l, i}^{\omega}>0$, $\gamma_{l, i}^{P}>0, \alpha_{l, i}^{\omega}>1, \alpha_{l, i}^{P}>1,0<\sigma_{l, i}^{\omega}<\frac{1}{1+\gamma_{l, i}^{\omega}}$ and $0<\sigma_{l, i}^{P}<\frac{1}{1+\gamma_{l, i}^{P}}$ as illustrated in Section 3. It should be mentioned that the parameters $\varepsilon^{\omega}$ and $\varepsilon^{P}$ are selected to be small enough here to obtain the allowable fluctuation ranges $\bar{\varepsilon}_{\omega}$ and $\bar{\varepsilon}_{P}$.

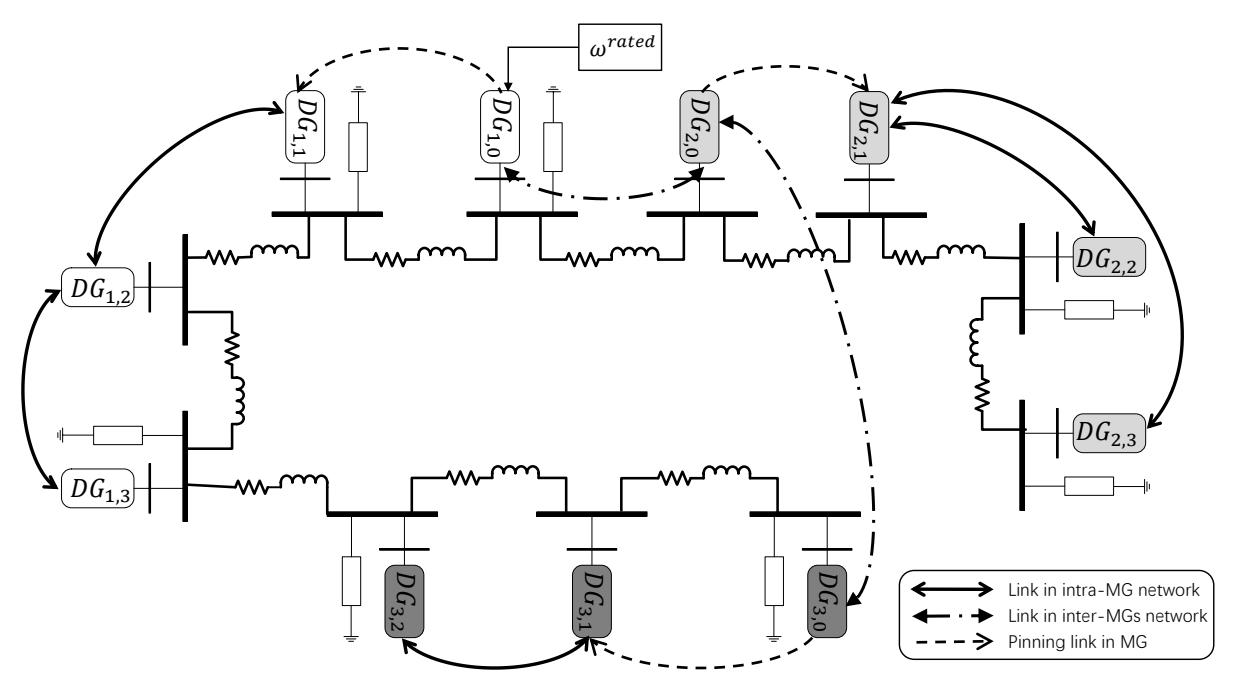

Figure 2. Test islanded MGs cluster.

The effectiveness in frequency restoration and active power sharing of the proposed control law will be verified by the scenarios of load changes and MG plug-and-play. Moreover, in order to demonstrate the superiority of hybrid-triggered mechanism in reducing communication burdens, the simulation result under periodic sampling mechanism is given to make a comparison. The control law based on periodic sampling mechanism for $t \in[k h,(k+1) h)$ is obtained as Formulas (57)-(60) by the discretization implementation of continuous information transmission in control law (5)-(8) according to the periodic sampling scheme.

$$
\begin{aligned}
& u_{l, 0}^{\omega}(t)=\sum_{m=1}^{s} \tilde{a}_{l m}\left(\omega_{m, 0}(k h)-\omega_{l, 0}(k h)\right)+\tilde{a}_{l 0}\left(\omega^{\text {rated }}-\omega_{l, 0}(k h)\right) \\
& u_{l, 0}^{P}(t)=\frac{1}{K_{l, 0}} \sum_{m=1}^{s} \tilde{a}_{l m}\left(K_{m, 0} P_{m, 0}(k h)-K_{l, 0} P_{l, 0}(k h)\right) \\
& u_{l, i}^{\omega}(t)=\sum_{i=1}^{n^{l}} a_{i j}^{l}\left(\omega_{l, j}(k h)-\omega_{l, i}(k h)\right)+a_{i 0}^{l}\left(\omega_{l, 0}(k h)-\omega_{l, i}(k h)\right) \\
& u_{l, i}^{P}(t)=\frac{1}{K_{l, i}}\left(\sum_{i=1}^{n^{l}} a_{i j}^{l}\left(K_{l, j} P_{l, j}(k h)-K_{l, i} P_{l, i}(k h)\right)+a_{i 0}^{l}\left(K_{l, 0} P_{l, 0}(k h)-K_{l, i} P_{l, i}(k h)\right)\right)
\end{aligned}
$$

where the non-negative integer $k$ is the sampling number and the constant $h$ is the fixed sampling period.

\subsection{Case A: Robustness against Load Changes}

In this case, the islanded MGs cluster is initially at the steady state with the total load power demand $182 \mathrm{~kW}$, and then the demand is increased to $275 \mathrm{~kW}$ at $5 \mathrm{~s}$, and finally returns to $182 \mathrm{~kW}$ at $15 \mathrm{~s}$. The control performance of the proposed hybrid-triggered control law is illustrated in Figures 3a and 4a. Figure 3a shows the response of frequency of all DGs in the islanded MGs cluster, and it implies that the frequency can be restored to the rated value when the load changes. Figure $4 \mathrm{a}$ 
shows that the active power output ratios of all the DGs achieve consensus with an acceptable fluctuation range, and this implies that the hybrid-triggered control law realizes the active power sharing. As mentioned, these ratios converge to $60 \%$ at $5 \mathrm{~s}$ and then return to $40 \%$ at $15 \mathrm{~s}$, which means that the active power generation and demand achieve a balance under load changes. These results verify the control performance of the proposed control law.

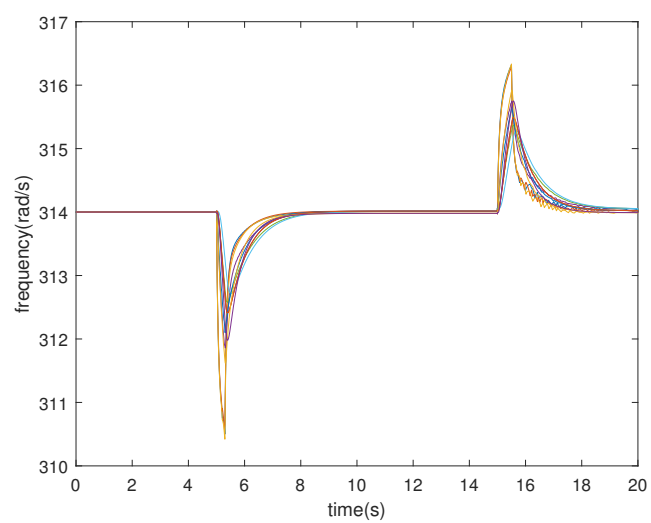

(a)

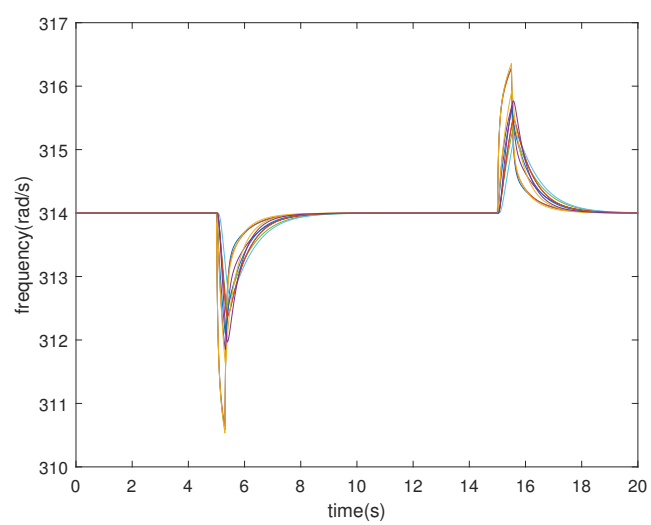

(b)

Figure 3. Control performance comparison in Case A. Frequency response under: (a): hybrid-triggered mechanism; (b): periodic sampling mechanism.

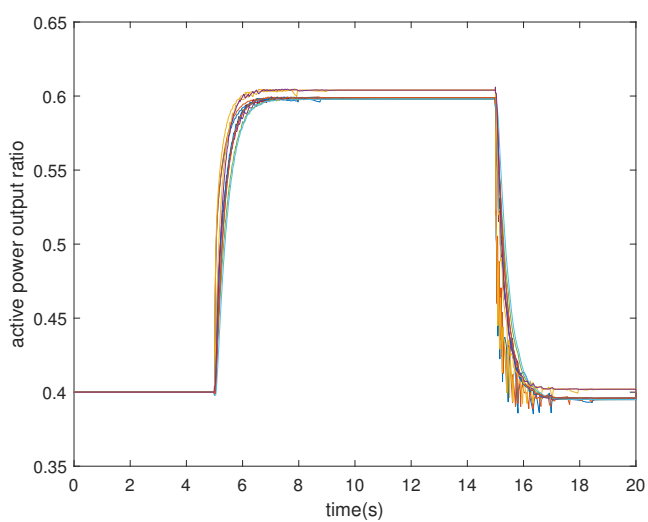

(a)

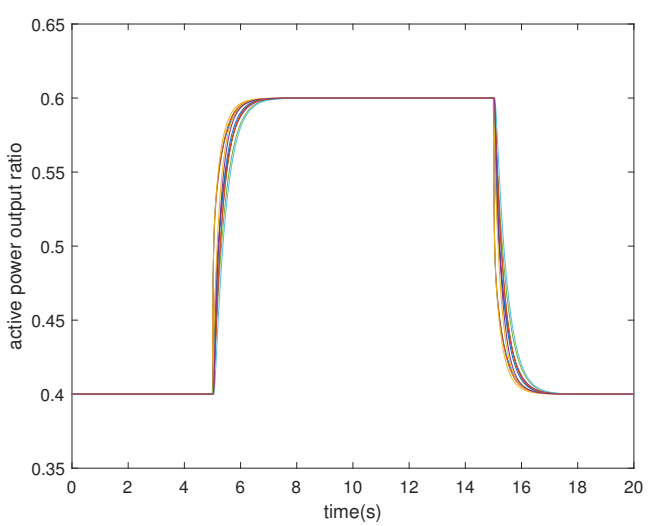

(b)

Figure 4. Control performance comparison in Case A. Active power output ratio response under: (a): hybrid-triggered mechanism; (b): periodic sampling mechanism.

Without loss of generality, the triggering time instants of $D G_{3,1}$ is given in Figure 5, where the abscissa and ordinate of circle signify the triggering time and the spending time since the last triggering, respectively. The unequal triggering periods demonstrate the demand-transmission character and the ability in adjusting communication frequency of a hybrid-triggered mechanism. 


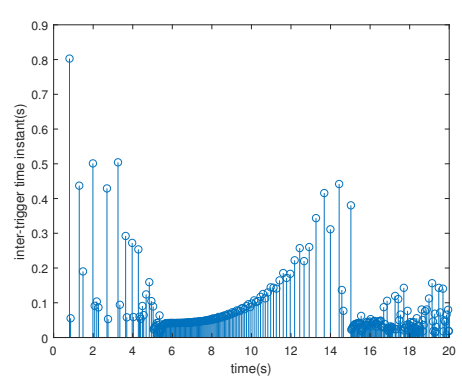

(a)

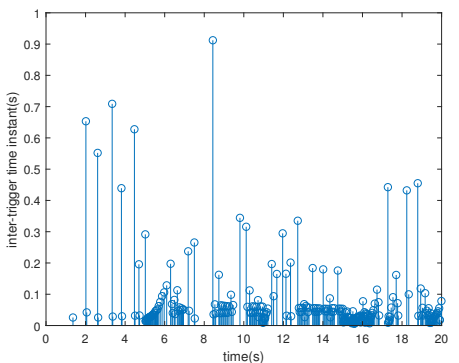

(b)

Figure 5. Triggering time instants of $D G_{3,1}$ under hybrid-triggered mechanism in Case A about: (a): frequency; (b): active power output ratio.

The simulation results under periodic sampled control law (57)-(60) are given here to make comparison. In order to obtain a similar control performance under hybrid-triggered control (13) and (14) and (40) and (41), the sampling period in control law (57)-(60) should be selected as $\mathrm{h}=0.02 \mathrm{~s}$. Figure $3 \mathrm{~b}$ and Figure $4 \mathrm{~b}$ illustrate the state response under the control law (57)-(60), and it can be seen from Figures 3 and 4 that nearly identical control performances can be ensured under the two types of mechanisms. However, focusing on the number of communication and sampling of each DG under the two types of mechanisms during time interval $[0,20]$ s given in Tables 1 and 2, we can get that the hybrid-triggered mechanism can lead to a lower number of sampling and communication than the periodic sampling one with the similar control performance. Figure 6 shows the comparison of communication number intuitively and illustrates the superiority of a hybrid-triggered mechanism in reducing communication burdens.

Table 1. Comparison of communication number between hybrid-triggered mechanism(HTM) and periodic sampling mechanism(PSM) about frequency in Case A.

\begin{tabular}{|c|c|c|c|c|c|c|c|c|c|c|c|}
\hline & $D G_{1,0}$ & $D G_{1,1}$ & $D G_{1,2}$ & $D G_{1,3}$ & $D G_{2,0}$ & $D G_{2,1}$ & $D G_{2,2}$ & $D G_{2,3}$ & $D G_{3,0}$ & $D G_{3,1}$ & $D G_{3,2}$ \\
\hline HTM & 560 & 610 & 557 & 197 & 336 & 496 & 412 & 248 & 410 & 240 & 383 \\
\hline PSM & 1000 & 1000 & 1000 & 1000 & 1000 & 1000 & 1000 & 1000 & 1000 & 1000 & 1000 \\
\hline rate & $56.0 \%$ & $61.0 \%$ & $55.7 \%$ & $19.7 \%$ & $33.6 \%$ & $49.6 \%$ & $41.2 \%$ & $24.8 \%$ & $41.0 \%$ & $24.0 \%$ & $38.3 \%$ \\
\hline
\end{tabular}

Table 2. Comparison of communication number between hybrid-triggered mechanism(HTM) and periodic sampling mechanism(PSM) about active power output ratio in Case A.

\begin{tabular}{|c|c|c|c|c|c|c|c|c|c|c|c|}
\hline & $D G_{1,0}$ & $D G_{1,1}$ & $D G_{1,2}$ & $D G_{1,3}$ & $D G_{2,0}$ & $D G_{2,1}$ & $D G_{2,2}$ & $D G_{2,3}$ & $D G_{3,0}$ & $D G_{3,1}$ & $D G_{3,2}$ \\
\hline HTM & 457 & 678 & 575 & 254 & 282 & 292 & 290 & 544 & 294 & 269 & 265 \\
\hline PSM & 1000 & 1000 & 1000 & 1000 & 1000 & 1000 & 1000 & 1000 & 1000 & 1000 & 1000 \\
\hline rate & $45.7 \%$ & $67.8 \%$ & $57.5 \%$ & $25.4 \%$ & $28.2 \%$ & $29.2 \%$ & $29.0 \%$ & $54.4 \%$ & $29.4 \%$ & $26.9 \%$ & $26.5 \%$ \\
\hline
\end{tabular}




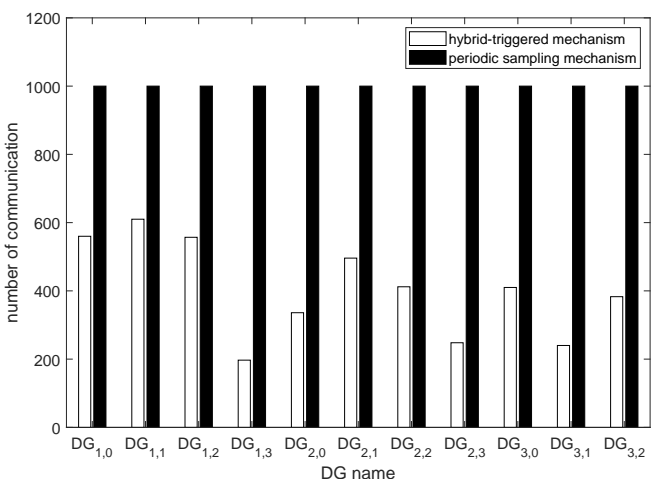

(a)

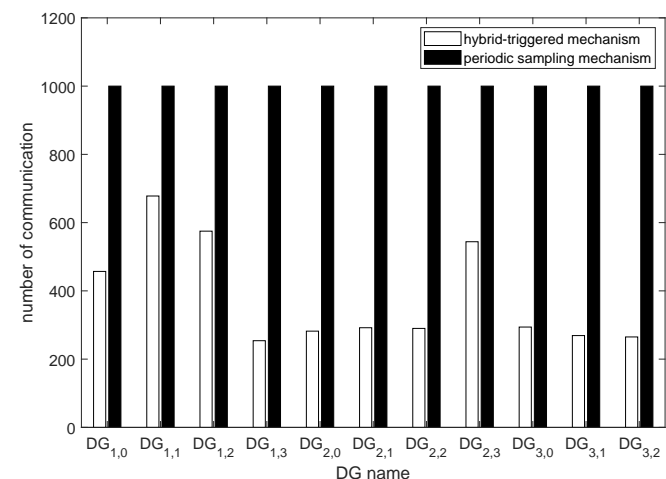

(b)

Figure 6. Communication number comparison between hybrid-triggered and periodic sampling mechanisms in Case A about: (a): frequency; (b): active power output ratio.

\subsection{Case B: MG Plug-and-Play Capability}

The islanded MGs cluster is initially at the steady state where each of the load power demand is $26 \mathrm{~kW}$ in this case, and then $M G_{3}$ is unplugged from the cluster at $5 \mathrm{~s}$, and finally it is plugged back into the cluster at $15 \mathrm{~s}$. The power and communication links connecting between $M G_{3}$ and the cluster are lost during the time interval $[5,15) \mathrm{s}$, and the test system is divided into two independent parts which are $M G_{3}$ and the remaining cluster containing $M G_{1}$ and $M G_{2}$. Meanwhile, all the DGs in $M G_{3}$ execute the intra-MG control law during $[5,15)$ s since the inter-MGs communication of $M G_{3}$ is nonexistent.

Figure 7a shows the response of frequency of all the DGs under the proposed hybrid-triggered distributed secondary control, which implies that the frequency can be restored to the rated value under the MG plug-and-play case. The response of active power output ratios of all the DGs under the hybrid-triggered control law is given in Figure 8a. It can be seen that the active power output ratios of DGs in $M G_{3}$ and the remaining cluster converge to $30 \%$ and $47 \%$, respectively, when $M G_{3}$ is plugged out at $5 \mathrm{~s}$, which demonstrates that the active power sharing in these two independent parts can be realized respectively with an acceptable fluctuation range. Moreover, all of these ratios converge to $40 \%$ when $M G_{3}$ is plugged back at 15 s and the initial steady state operating mode is formed again. These results verify the control performance of the proposed hybrid-triggered control law under the MG plug-and-play case. The triggering time instant given in Figure 9 implies the demand-transmission character of hybrid-triggered mechanism.

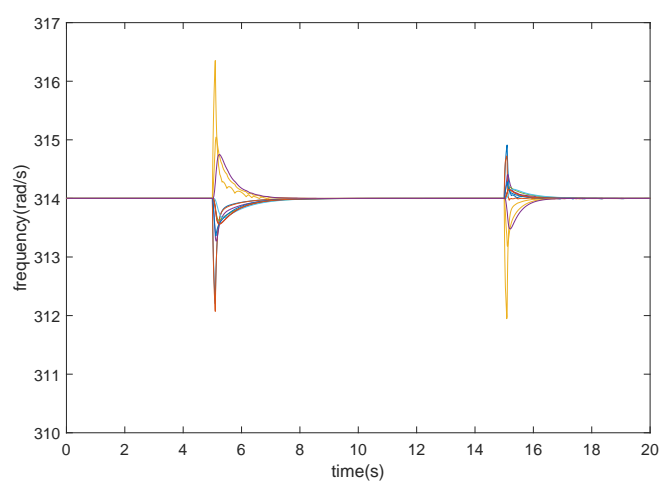

(a)

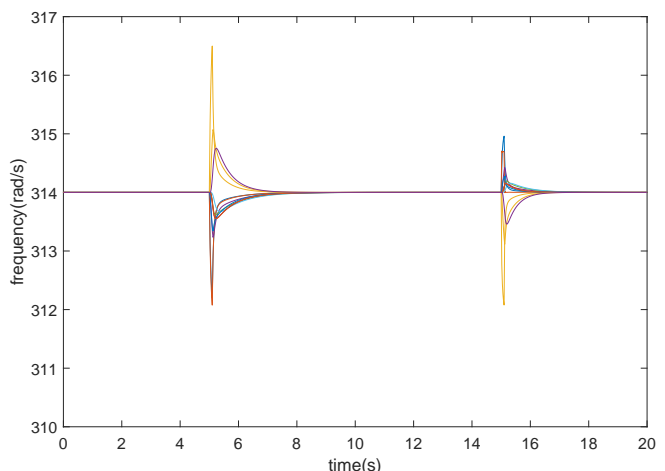

(b)

Figure 7. Control performance comparison in Case B. Frequency response under: (a): hybrid-triggered mechanism; (b): periodic sampling mechanism. 


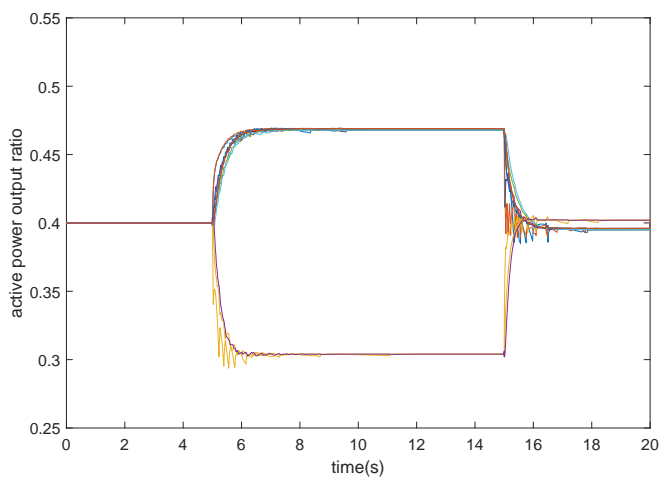

(a)

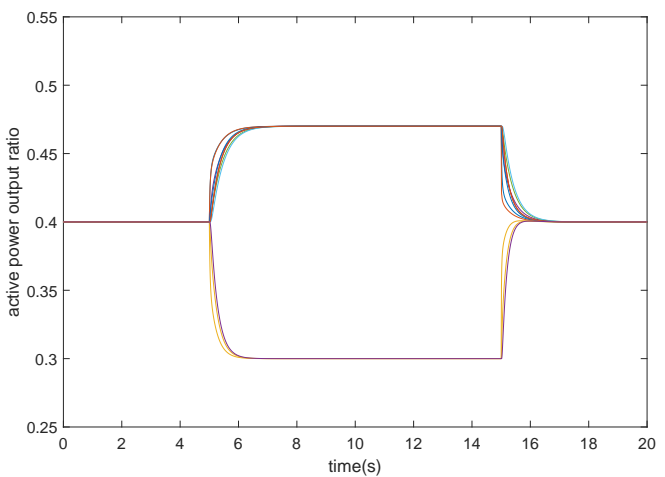

(b)

Figure 8. Control performance comparison in Case B. Active power output ratio response under: (a): hybrid-triggered mechanism; (b): periodic sampling mechanism.

Similar to Case A, the system response under periodic sampling mechanism based control law (57)-(60) with the fixed period $\mathrm{h}=0.02 \mathrm{~s}$ is shown in Figures $7 \mathrm{~b}$ and $8 \mathrm{~b}$. The comparisons in Figures 7 and 8 show the similar control performance under the two types of mechanisms. However, the comparisons of communication and sampling number between the two types of mechanisms given in Figure 10 and Tables 3 and 4 represent the communication burdens that can be reduced further under the proposed hybrid-triggered mechanism.

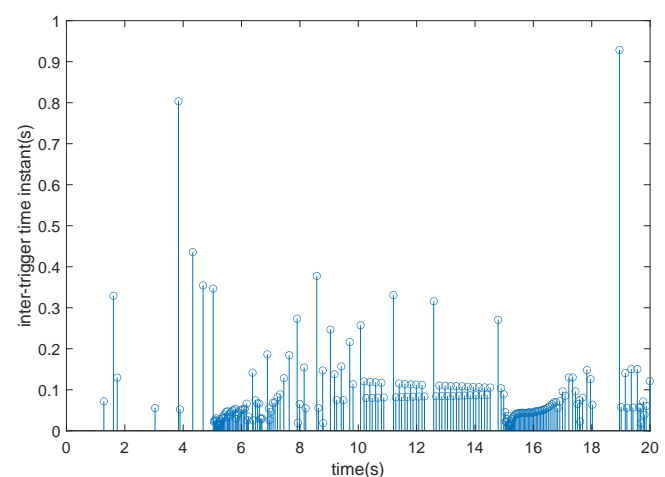

(a)

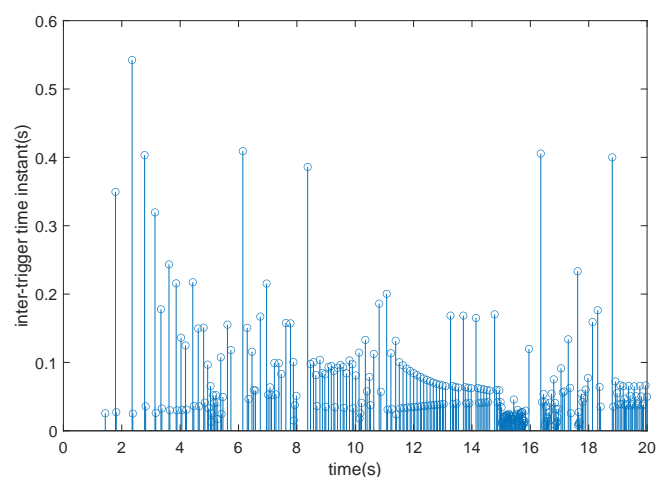

(b)

Figure 9. Triggering time instants of $D G_{3,1}$ under hybrid-triggered mechanism in Case B about: (a): frequency; (b): active power output ratio.

Table 3. Comparison of communication number between hybrid-triggered mechanism(HTM) and periodic sampling mechanism(PSM) about frequency in Case B.

\begin{tabular}{|c|c|c|c|c|c|c|c|c|c|c|c|}
\hline & $D G_{1,0}$ & $D G_{1,1}$ & $D G_{1,2}$ & $D G_{1,3}$ & $D G_{2,0}$ & $D G_{2,1}$ & $D G_{2,2}$ & $D G_{2,3}$ & $D G_{3,0}$ & $D G_{3,1}$ & $D G_{3,2}$ \\
\hline HTM & 343 & 200 & 318 & 356 & 263 & 287 & 259 & 197 & 192 & 277 & 369 \\
\hline PSM & 1000 & 1000 & 1000 & 1000 & 1000 & 1000 & 1000 & 1000 & 1000 & 1000 & 1000 \\
\hline rate & $34.3 \%$ & $20.0 \%$ & $31.8 \%$ & $35.6 \%$ & $26.3 \%$ & $28.7 \%$ & $25.9 \%$ & $19.7 \%$ & $19.2 \%$ & $27.7 \%$ & $36.9 \%$ \\
\hline
\end{tabular}


Table 4. Comparison of communication number between hybrid-triggered mechanism(HTM) and periodic sampling mechanism(PSM) about active power output ratio in Case B.

\begin{tabular}{|c|c|c|c|c|c|c|c|c|c|c|c|}
\hline & $D G_{1,0}$ & $D G_{1,1}$ & $D G_{1,2}$ & $D G_{1,3}$ & $D G_{2,0}$ & $D G_{2,1}$ & $D G_{2,2}$ & $D G_{2,3}$ & $D G_{3,0}$ & $D G_{3,1}$ & $D G_{3,2}$ \\
\hline HTM & 233 & 262 & 243 & 250 & 227 & 335 & 232 & 267 & 173 & 267 & 320 \\
\hline PSM & 1000 & 1000 & 1000 & 1000 & 1000 & 1000 & 1000 & 1000 & 1000 & 1000 & 1000 \\
\hline rate & $23.3 \%$ & $26.2 \%$ & $24.3 \%$ & $25.0 \%$ & $22.7 \%$ & $33.5 \%$ & $23.2 \%$ & $26.7 \%$ & $17.3 \%$ & $26.7 \%$ & $32.0 \%$ \\
\hline
\end{tabular}

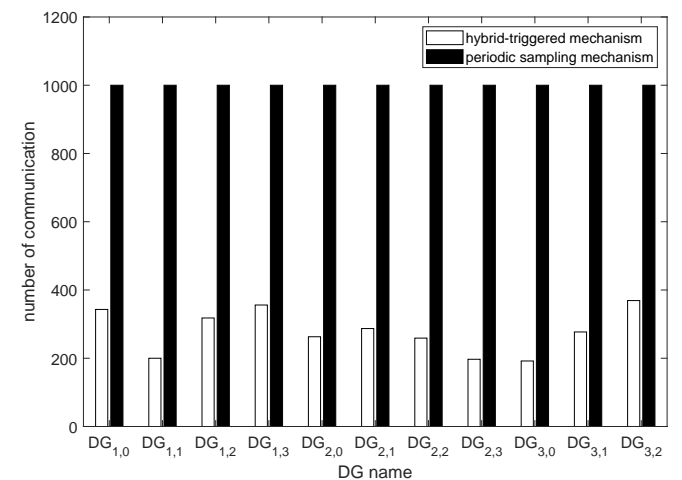

(a)

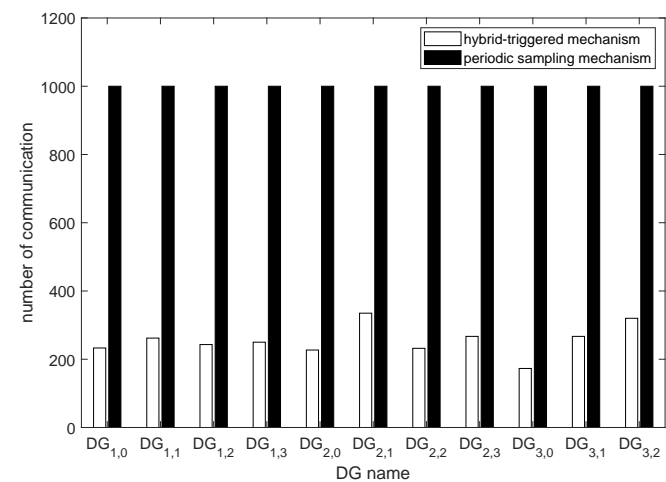

(b)

Figure 10. Communication number comparison between hybrid-triggered and periodic sampling mechanisms in Case B about: (a): frequency; (b): active power output ratio.

\section{Conclusions}

This paper studies the distributed secondary control of islanded MGs cluster, which contains the inter-MGs and intra-MG control law. The control aims to restore the frequency to the rated value and realize the active power sharing of all DGs in the cluster. Considering the communication resource limitation, the hybrid-triggered mechanism, which contains the self-triggered mechanism for inter-MGs information transmission and the event-triggered mechanism for intra-MG one, is introduced in the distributed secondary control of islanded MGs cluster. Since the period of information transmission is adjusted adaptively according to the system's state under hybrid-triggered mechanism, the communication burdens can be sharply reduced. The simulation results verify the control performance and the superiority in reducing communication burdens of the proposed hybrid-triggered control. Our future work will study the resilient control method based on triggered mechanism for islanded MG cluster under cyber-attack.

Author Contributions: S.W. studied the methodology and implemented the experimental simulation. Y.X., J.L., and Y.L. formulated the microgrid model and control purpose. All authors have read and agreed to the published version of the manuscript.

Funding: This research was sponsored by the Found of NARI Technology Co., Ltd. Nanjing, China (2019out129), Project funded by the China Postdoctoral Science Foundation (2018M642294) and Jiangsu Planned Projects for Postdoctoral Research Funds (2018K006A).

Conflicts of Interest: The authors declare no conflict of interest.

\section{References}

1. Katiraei, F.; Iravani, M.R. Power management strategies for a microgrid with multiple distributed generation units. IEEE Trans. Power Syst. 2006, 21, 1821-1831. [CrossRef]

2. Liu, S.; Liu, P.X.; Wang, X. Stochastic small-signal stability analysis of grid-connected photovoltaic systems. IEEE Trans. Ind. Electron. 2015, 63, 1027-1038. [CrossRef] 
3. Pogaku, N.; Prodanovic, M.; Green, T.C. Modeling, analysis and testing of autonomous operation of an inverter-based microgrid. IEEE Trans. Power Electron. 2007, 22, 613-625. [CrossRef]

4. Atawi, I.E.; Kassem, A.M.; Zaid, S.A. Modeling, Management, and Control of an Autonomous Wind/Fuel Cell Micro-Grid System. Processes 2019, 7, 85. [CrossRef]

5. Lai, J.; Lu, X.; Yu, X.; Monti, A. Cluster-oriented distributed cooperative control for multiple AC microgrids. IEEE Trans. Ind. Inf. 2019, 15, 5906-5918. [CrossRef]

6. Liu, K.; Liu, T.; Tang, Z.; Hill, D.J. Distributed MPC-Based Frequency Control in Networked Microgrids With Voltage Constraints. IEEE Trans. Smart Grid 2019, 10, 6343-6354. [CrossRef]

7. Du, Y.; Lu, X.; Wang, J.; Lukic, S. Distributed Secondary Control Strategy for Microgrid Operation with Dynamic Boundaries. IEEE Trans. Smart Grid 2019, 10, 5269-5282. [CrossRef]

8. Yan, Y.; Shi, D.; Bian, D.; Huang, B.; Yi, Z.; Wang, Z. Small-Signal Stability Analysis and Performance Evaluation of Microgrids Under Distributed Control. IEEE Trans. Smart Grid 2019, 10, 4848-4858. [CrossRef]

9. Lu, X.; Yu, X.; Lai, J.; Wang, Y.; Guerrero, J.M. A Novel Distributed Secondary Coordination Control Approach for Islanded Microgrids. IEEE Trans. Smart Grid 2018, 9, 2726-2740. [CrossRef]

10. Zholbaryssov, M.; Dominguez-Garcia, A.D. Microgrid Distributed Frequency Control Over Time-Varying Communication Networks. In Proceedings of the 2018 IEEE Conference on Decision and Control (CDC), Miami Beach, FL, USA, 17-19 December 2018; pp. 5722-5727 [CrossRef]

11. Lu, X.; Lai, J.; Yu, X.; Wang, Y.; Guerrero, J.M. Distributed coordination of islanded microgrid clusters using a two-layer intermittent communication network. IEEE Trans. Ind. Inf. 2017, 14, 3956-3969. [CrossRef]

12. Maknouninejad, A.; Qu, Z.; Enslin, J.; Kutkut, N. Clustering and cooperative control of distributed generators for maintaining microgrid unified voltage profile and complex power control. In PES TED 2012; IEEE: Piscataway, NJ, USA, 2012; pp. 1-8.

13. Nutkani, I.U.; Loh, P.C.; Blaabjerg, F. Distributed operation of interlinked AC microgrids with dynamic active and reactive power tuning. IEEE Trans. Ind. Appl. 2013, 49, 2188-2196. [CrossRef]

14. Lai, J.; Zhou, H.; Lu, X.; Yu, X.; Hu, W. Droop-based distributed cooperative control for microgrids with time-varying delays. IEEE Trans. Smart Grid 2016, 7, 1775-1789. [CrossRef]

15. Yue, D.; Tian, E.; Han, Q.L. A Delay System Method for Designing Event-Triggered Controllers of Networked Control Systems. IEEE Trans. Autom. Control 2013, 58, 475-481. [CrossRef]

16. Wang, X.; Lemmon, M. Event-Triggering in Distributed Networked Control Systems. IEEE Trans. Autom. Control 2011, 56, 586-601. [CrossRef]

17. Seyboth, G.S.; Dimarogonas, D.V.; Johansson, K.H. Event-based broadcasting for multi-agent average consensus. Automatica 2013, 49, 245-252. [CrossRef]

18. Almeida, J.; Silvestre, C.; Pascoal, A. Synchronization of multiagent systems using event-triggered and self-triggered broadcasts. IEEE Trans. Autom. Control 2017, 62, 4741-4746. [CrossRef]

19. Ding, L.; Wang, L.Y.; Yin, G.; Zheng, W.X.; Han, Q. Distributed Energy Management for Smart Grids With an Event-Triggered Communication Scheme. IEEE Trans. Control Syst. Technol. 2018, 14, 1950-1961. [CrossRef]

20. Chen, M.; Xiao, X.; Guerrero, J.M. Secondary Restoration Control of Islanded Microgrids With a Decentralized Event-Triggered Strategy. IEEE Trans. Ind. Inf. 2018, 14, 3870-3880. [CrossRef]

21. Li, C.; Yu, X.; Yu, W.; Huang, T.; Liu, Z.W. Distributed event-triggered scheme for economic dispatch in smart grids. IEEE Trans. Ind. Inf. 2016, 12, 1775-1785. [CrossRef]

22. Fan, Y.; Hu, G.; Egerstedt, M. Distributed reactive power sharing control for microgrids with event-triggered communication. IEEE Trans. Control Syst. Technol. 2016, 25, 118-128. [CrossRef]

23. Xu, S.; Sun, H.; Zhao, B.; Yi, J.; Weng, S.; Chen, J.; Dou, C. Distributed Optimal Frequency Regulation for Multiple Distributed Power Generations with an Event-Triggered Communication Mechanism. Processes 2020, 8, 169. [CrossRef]

24. Fan, Y.; Zhang, C.; Song, C. Sampling-based self-triggered coordination control for multi-agent systems with application to distributed generators. Int. J. Syst. Sci. 2018, 49, 3048-3062. [CrossRef]

25. Tahir, M.; Mazumder, S.K. Self-triggered communication enabled control of distributed generation in microgrids. IEEE Trans. Ind. Inf. 2015, 11, 441-449. [CrossRef] 
26. Olfati-Saber, R.; Murray, R.M. Consensus problems in networks of agents with switching topology and time-delays. IEEE Trans. Autom. Control 2004, 49, 1520-1533. [CrossRef]

27. Hong, Y.; Hu, J.; Gao, L. Tracking control for multi-agent consensus with an active leader and variable topology. Automatica 2006, 42, 1177-1182. [CrossRef]

(C) 2020 by the authors. Licensee MDPI, Basel, Switzerland. This article is an open access article distributed under the terms and conditions of the Creative Commons Attribution (CC BY) license (http://creativecommons.org/licenses/by/4.0/). 